\title{
A Study of Non-structure Cohesion in the Texts in New Senior English for China Student's Book 5 and 6
}

\author{
Yuan Zhao \\ Shanxi Normal University, China
}

\begin{abstract}
Since the publication of Cohesive in English co-authored by Halliday and Hasan in 1976, more linguistics at home and abroad have been analyzing and studying this theory in detail. Thus, cohesion theory has been steadily growing. Existing studies, however, tended to focus on the analysis of cohesive devices in a variety of discourses other than the texts from English textbooks for Chinese high school learners. To address this gap, this study used 5 articles from New Senior English for China Student's Book 5 and 6 to explore the non-structure cohesive devices. By means of the coding scheme suggested by Halliday and Hasan in 1976, the cohesive patterns in a text were represented. The results showed that coherence in the 5 texts largely depends on lexical cohesion. Then, a further analysis of the five lexical cohesive devices showed that among them, same item having reference that is identical proved to be the most in the texts. The main reasons is perhaps that since the texts are from school textbooks for Chinese teenagers, the student readers almost certainly do not have the ability to make much sense of a fairly complex text whose coherence may largely depends on substitution or ellipsis.
\end{abstract}

Index Terms - cohesive patterns, non-structure cohesion, text

\section{INTRODUCTION}

Cohesion refers to the linguistic patterns by which the speaker can signal the experiential and interpersonal coherence of the text - and is thus a textual phenomenon - we can point to features of the text which serve a cohesive function (Geoff Thompson, 2008). Cohesion occurs where the interpretation of some element in the discourse is dependent on that of another (Halliday \& Hasan, 1976). In Language, Context and Text published in 1985 by Halliday and Hasan, Hasan expanded the covering range of the concept of cohesion, which is divided into structure cohesion and non-structure cohesion. The former one includes parallel structure, theme-rheme structure, given information —new information structure. The latter is made up of reference, substitution, ellipsis, lexical cohesion and conjunction.

The five types of non-structure cohesion have a great impact on textual research at home and abroad. Our intention in this paper is to survey the lexicogrammatical resources in some of the reading texts and analyse the cohesive patterns in them, through which a student reader is able to process a text, thus interpret it and determine how he does so.

\section{ANALYSIS OF NON-STRUCTURE COHESION IN TEXTS}

The coding scheme suggested by Halliday and Hasan in 1976 provides a means of representing the cohesive patterns in a text, that is, reference, substitution, ellipsis, conjunction and lexical cohesion. Besides, they also provided us with a term - a tie - to refer to a single instance of cohesion, a term for one occurrence of a pair of cohesively related items.

For the text, each sentence is given an index number, and the total number of ties in that sentence is entered in the appropriate column. Then for each tie we specify the type of cohesion and its distance and direction (Halliday \& Hasan, 1976).

TABLE 2-1

THE ANALYSIS OF COHESION IN TEXT 1

\begin{tabular}{|c|c|c|c|c|c|}
\hline $\begin{array}{l}\text { Sentence } \\
\text { number }\end{array}$ & $\begin{array}{c}\text { No. of } \\
\text { ties }\end{array}$ & Cohesive item & Type & Distance & Presupposed item \\
\hline \multirow[t]{4}{*}{2} & 4 & But & $\mathrm{C} 23.1$ & 0 & $(\mathrm{~S} .1)$ \\
\hline & & he $(2 x)$ & R11.6 & 0 & John Snow \\
\hline & & help & L5 & 0 & attended \\
\hline & & ordinary people & L5 & 0 & Queen Victoria \\
\hline \multirow[t]{3}{*}{3} & 3 & This & R21.6 & 0 & cholera \\
\hline & & disease & L3.6 & 0 & cholera \\
\hline & & its & $\mathrm{R} 13.8$ & 0 & cholera \\
\hline 4 & 1 & its $(2 x)$ & $\mathrm{R} 13.8$ & 0 & cholera \\
\hline \multirow[t]{3}{*}{5} & 3 & So & C31.1 & 0 & (S.4) \\
\hline & & people & L1.6 & N.2 & people \\
\hline & & die & L5 & N.1 & deadly \\
\hline
\end{tabular}




\begin{tabular}{|c|c|c|c|c|c|}
\hline 6 & 4 & $\begin{array}{c}\text { John Snow } \\
\text { wanted } \\
\text { face/solve } \\
\text { challenge/problem }\end{array}$ & $\begin{array}{l}\text { L1.6 } \\
\text { L5 } \\
\text { L5 } \\
\text { L5 }\end{array}$ & $\begin{array}{c}\text { N.4 } \\
\text { N.3 } \\
\text { N.3 } \\
0\end{array}$ & $\begin{array}{c}\text { John Snow } \\
\text { inspired } \\
\text { help } \\
\text { outbreak }\end{array}$ \\
\hline 7 & 4 & $\begin{array}{c}\mathrm{He} \\
\text { cholera } \\
\text { controlled/ found } \\
\text { cause }\end{array}$ & $\begin{array}{l}\text { R11.6 } \\
\text { L1.6 } \\
\text { L5 } \\
\text { L1.6 }\end{array}$ & $\begin{array}{c}0 \\
\text { N.4 } \\
\text { N.2 } \\
\text { N.2 }\end{array}$ & $\begin{array}{l}\text { John Snow } \\
\text { cholera } \\
\text { understood } \\
\text { cause }\end{array}$ \\
\hline 8 & 4 & $\begin{array}{c}\mathrm{He} \\
\text { interested } \\
\text { cholera } \\
\text { people }\end{array}$ & $\begin{array}{l}\text { R11.6 } \\
\text { L5 } \\
\text { L1.6 } \\
\text { L1.6 }\end{array}$ & $\begin{array}{c}\text { M.1 } \\
\text { N.1 } \\
0 \\
\text { N.2 }\end{array}$ & $\begin{array}{c}\mathrm{He} \rightarrow \text { John Snow } \\
\text { wanted } \\
\text { cholera } \\
\text { people }\end{array}$ \\
\hline 9 & 3 & $\begin{array}{l}\text { The first } \\
\text { suggested } \\
\text { cholera }\end{array}$ & $\begin{array}{c}\text { E12.1 } \\
\text { L5 } \\
\text { L1.6 }\end{array}$ & $\begin{array}{l}0 \\
0 \\
0 \\
\end{array}$ & $\begin{array}{c}\text { two theories } \\
\text { explained } \\
\text { cholera }\end{array}$ \\
\hline 10 & 4 & $\begin{array}{c}\text { gas } \\
\text { it } \\
\text { its } \\
\text { victims }\end{array}$ & $\begin{array}{c}\text { L2.6 } \\
\text { R13.6 } \\
\text { R13.8 } \\
\text { L2.6 }\end{array}$ & $\begin{array}{c}0 \\
0 \\
0 \\
\text { N.1 }\end{array}$ & $\begin{array}{c}\text { air } \\
\text { cholera } \\
\text { cholera } \\
\text { people }\end{array}$ \\
\hline 11 & 5 & $\begin{array}{l}\text { The second } \\
\text { suggested } \\
\text { people } \\
\text { this } \\
\text { disease } \\
\end{array}$ & $\begin{array}{c}\text { E12.1 } \\
\text { L1.6 } \\
\text { L1.8 } \\
\text { R21.6 } \\
\text { L3.6 }\end{array}$ & $\begin{array}{c}\text { N.1 } \\
\text { N.1 } \\
0 \\
\text { N.1 } \\
\text { N.1 }\end{array}$ & $\begin{array}{l}\text { two theories } \\
\text { suggested } \\
\text { victims } \\
\text { cholera } \\
\text { cholera }\end{array}$ \\
\hline 12 & 7 & $\begin{array}{l}\text { stomach } \\
\text { the }\end{array}$ & $\begin{array}{c}\text { L2.7 } \\
\text { R23.6 }\end{array}$ & $\begin{array}{l}0 \\
0\end{array}$ & $\begin{array}{c}\text { bodies } \\
\text { this disease }\end{array}$ \\
\hline $\begin{array}{l}\text { Sentence } \\
\text { number }\end{array}$ & $\begin{array}{c}\text { No. of } \\
\text { ties }\end{array}$ & Cohesive item & Type & Distance & Presupposed item \\
\hline & & $\begin{array}{l}\text { disease } \\
\text { body } \\
\text { affected } \\
\text { person } \\
\text { died }\end{array}$ & $\begin{array}{l}\text { L1.6 } \\
\text { L1.6 } \\
\text { L5 } \\
\text { L2.6 } \\
\text { L5 }\end{array}$ & $\begin{array}{c}0 \\
0 \\
\text { N.9 } \\
0 \\
\text { N.3 }\end{array}$ & $\begin{array}{c}\text { disease } \\
\text { bodies } \\
\text { exposed } \\
\text { people } \\
\text { killed }\end{array}$ \\
\hline 13 & 3 & $\begin{array}{c}\text { John Snow } \\
\text { the second } \\
\text { theory }\end{array}$ & $\begin{array}{c}\text { L1.6 } \\
\text { R34.7 } \\
\text { L1.6 } \\
\end{array}$ & $\begin{array}{l}\text { N.6 } \\
\text { M.1 } \\
\text { N.4 } \\
\end{array}$ & $\begin{array}{c}\text { John Snow } \\
\text { the second } \rightarrow \text { two theories } \\
\text { theories }\end{array}$ \\
\hline 14 & 8 & $\begin{array}{c}\text { So } \\
\text { another } \\
\text { outbreak } \\
\text { hit } \\
\text { London } \\
\text { in1854 } \\
\text { he } \\
\text { ready }\end{array}$ & $\begin{array}{l}\text { C31.1 } \\
\text { R33.6 } \\
\text { L1.8 } \\
\text { L2.6 } \\
\text { L1.6 } \\
\text { L5 } \\
\text { R11.6 } \\
\text { L5 }\end{array}$ & $\begin{array}{c}0 \\
\text { N.8 } \\
\text { N.8 } \\
\text { N.1 } \\
\text { N.12 } \\
\text { N.10 } \\
0 \\
\text { N.5 }\end{array}$ & $\begin{array}{c}\text { (S.13) } \\
\text { an outbreak(S.5) } \\
\text { an outbreak(S.5) } \\
\text { attacked } \\
\text { London(S.1) } \\
\text { its day } \\
\text { John Snow } \\
\text { interested }\end{array}$ \\
\hline 15 & 7 & $\begin{array}{c}\text { the } \\
\text { disease } \\
\text { spread } \\
\text { quickly } \\
\text { poor } \\
\text { he } \\
\text { information }\end{array}$ & $\begin{array}{l}\text { R23.6 } \\
\text { L1.6 } \\
\text { L5 } \\
\text { L1.9 } \\
\text { L5 } \\
\text { R11.6 } \\
\text { L5 }\end{array}$ & $\begin{array}{c}\text { N.2 } \\
\text { N.2 } \\
0 \\
\text { N.2 } \\
\text { N.12 } \\
\text { M.1 } \\
0\end{array}$ & $\begin{array}{c}\text { the disease } \\
\text { disease } \\
\text { hit } \\
\text { quickly } \\
\text { ordinary } \\
\text { he } \rightarrow \text { John Snow } \\
\text { enquiry }\end{array}$ \\
\hline 16 & 6 & $\begin{array}{c}\text { in two particular streets } \\
\text { cholera } \\
\text { outbreak } \\
\text { people } \\
\text { died }\end{array}$ & $\begin{array}{c}\text { L5 } \\
\text { L2.6 } \\
\text { L1.6 } \\
\text { L1.7 } \\
\text { L1.6 }\end{array}$ & $\begin{array}{c}0 \\
0 \\
\text { N.1 } \\
\text { N.4 } \\
\text { N.3 }\end{array}$ & $\begin{array}{c}\text { neighbourhoods } \\
\text { disease } \\
\text { another outbreak } \\
\text { people } \\
\text { died }\end{array}$ \\
\hline 17 & 3 & $\begin{array}{c}\mathrm{He} \\
\text { find out } \\
\text { why } \\
\end{array}$ & $\begin{array}{l}\text { R11.6 } \\
\text { L1.6 } \\
\text { L5 } \\
\end{array}$ & $\begin{array}{l}\text { M.2 } \\
\text { N.9 } \\
\text { N.9 } \\
\end{array}$ & $\begin{array}{c}\text { he } \rightarrow \text { John Snow } \\
\text { found } \\
\text { cause }\end{array}$ \\
\hline 18 & 4 & $\begin{array}{c}\text { First } \\
\text { he } \\
\text { dead } \\
\text { people }\end{array}$ & $\begin{array}{l}\text { C43.1 } \\
\text { R11.6 } \\
\text { L5 } \\
\text { L1.6 } \\
\end{array}$ & $\begin{array}{c}0 \\
\text { M.3 } \\
\text { N.1 } \\
\text { N.1 }\end{array}$ & $\begin{array}{c}\text { S.17 }) \\
\mathrm{He} \rightarrow \text { John Snow } \\
\text { died } \\
\text { people }\end{array}$ \\
\hline 19 & 4 & $\begin{array}{c}\text { This } \\
\text { him } \\
\text { cause } \\
\text { disease }\end{array}$ & $\begin{array}{l}\text { R21.6 } \\
\text { R11.6 } \\
\text { L5 } \\
\text { L3.6 }\end{array}$ & $\begin{array}{c}0 \\
\text { M.4 } \\
0 \\
\text { N.2 }\end{array}$ & $\begin{array}{c}\text { (S.18) } \\
\text { he } \rightarrow \text { John Snow } \\
\text { why } \\
\text { cholera }\end{array}$ \\
\hline 20 & 2 & deaths & L5 & 0 & dead \\
\hline \multirow[t]{2}{*}{$\begin{array}{c}\text { Sentence } \\
\text { number }\end{array}$} & $\begin{array}{c}\text { No. of } \\
\text { ties }\end{array}$ & Cohesive item & Type & Distance & Presupposed item \\
\hline & & Broad Street & L5 & N.1 & places/where \\
\hline
\end{tabular}




\begin{tabular}{|c|c|c|c|c|c|}
\hline 21 & 4 & $\begin{array}{c}\mathrm{He} \\
\text { also } \\
\text { houses } \\
\text { deaths } \\
\end{array}$ & $\begin{array}{l}\text { R11.6 } \\
\text { C11.1 } \\
\text { L5 } \\
\text { L1.8 } \\
\end{array}$ & $\begin{array}{c}\text { M.5 } \\
0 \\
0 \\
0\end{array}$ & $\begin{array}{c}\text { him } \rightarrow \text { John Snow } \\
\text { (S.20) } \\
\text { Broad Street } \\
\text { deaths }\end{array}$ \\
\hline 22 & 3 & $\begin{array}{c}\mathrm{He}(2 \times) \\
\text { this } \\
\text { investigations }\end{array}$ & $\begin{array}{c}\text { R11.6 } \\
\text { R21.6 } \\
\text { L5 } \\
\end{array}$ & $\begin{array}{c}\text { M.6 } \\
0 \\
\text { N.6 } \\
\end{array}$ & $\begin{array}{c}\mathrm{He} \rightarrow \text { John Snow } \\
(\mathrm{S} .21) \\
\text { information }\end{array}$ \\
\hline 23 & 5 & $\begin{array}{c}\text { He } \\
\text { discovered } \\
\text { these } \\
\text { people } \\
7 \text { Cambridge Street }\end{array}$ & $\begin{array}{l}\text { R11.6 } \\
\text { L5 } \\
\text { R21.6 } \\
\text { L4.6 } \\
\text { L5 }\end{array}$ & $\begin{array}{c}\text { M.7 } \\
0 \\
\text { N.1 } \\
\text { N.1 } \\
\text { N.2 }\end{array}$ & $\begin{array}{c}\mathrm{He} \rightarrow \text { John Snow } \\
\text { investigations } \\
\text { no deaths } \\
\text { no deaths } \\
\text { Broad Street }\end{array}$ \\
\hline 24 & 4 & $\begin{array}{c}\text { They } \\
\text { beer } \\
\text { water } \\
\text { pump } \\
\end{array}$ & $\begin{array}{l}\text { R14.6 } \\
\text { L5 } \\
\text { L1.6 } \\
\text { L1.6 } \\
\end{array}$ & $\begin{array}{c}0 \\
0 \\
\text { N.3 } \\
\text { N.3 } \\
\end{array}$ & $\begin{array}{c}\text { people } \\
\text { pub } \\
\text { water } \\
\text { pump } \\
\end{array}$ \\
\hline 25 & 3 & $\begin{array}{c}\text { the } \\
\text { water } \\
\text { was to blame }\end{array}$ & $\begin{array}{c}\text { R23.6 } \\
\text { L1.6 } \\
\text { L5 } \\
\end{array}$ & $\begin{array}{c}0 \\
0 \\
\text { N.5 } \\
\end{array}$ & $\begin{array}{l}\text { the water } \\
\text { the water } \\
\text { cause }\end{array}$ \\
\hline 26 & 7 & $\begin{array}{c}\text { Next } \\
\text { John Snow } \\
\text { looked into } \\
\text { the } \\
\text { water } \\
\text { these } \\
\text { streets } \\
\end{array}$ & $\begin{array}{c}\text { C41.1 } \\
\text { L1.6 } \\
\text { L5 } \\
\text { R23.6 } \\
\text { L1.6 } \\
\text { R21.6 } \\
\text { L1.7 } \\
\end{array}$ & $\begin{array}{c}0 \\
\mathrm{~N} .12 \\
\mathrm{~N} .2 \\
0 \\
0 \\
\mathrm{~N} .2 \\
\mathrm{~N} .2\end{array}$ & $\begin{array}{c}\text { (S.25) } \\
\text { John Snow } \\
\text { discovered } \\
\text { the water } \\
\text { the water } \\
7 \text { Cambridge Street } \\
7 \text { Cambridge Street }\end{array}$ \\
\hline 27 & 5 & $\begin{array}{c}\mathrm{He} \\
\text { found } \\
\text { it } \\
\text { water } \\
\text { London }\end{array}$ & $\begin{array}{l}\text { R11.6 } \\
\text { L5 } \\
\text { R13.6 } \\
\text { L1.8 } \\
\text { L1.6 }\end{array}$ & $\begin{array}{c}0 \\
0 \\
0 \\
0 \\
\text { N.12 }\end{array}$ & $\begin{array}{c}\text { John Snow } \\
\text { looked into } \\
\text { source } \\
\text { water } \\
\text { London }\end{array}$ \\
\hline 28 & 5 & $\begin{array}{c}\mathrm{He} \\
\text { astonished } \\
\text { people } \\
\text { Broad Street } \\
\text { pump } \\
\end{array}$ & $\begin{array}{l}\text { R11.6 } \\
\text { L2.6 } \\
\text { L1.7 } \\
\text { L1.6 } \\
\text { L1.6 }\end{array}$ & $\begin{array}{l}\text { M.1 } \\
\text { N.22 } \\
\text { N.4 } \\
\text { N.7 } \\
\text { N.3 } \\
\end{array}$ & $\begin{array}{c}\mathrm{He} \rightarrow \text { John Snow } \\
\text { terrified } \\
\text { people } \\
\text { Broad Street } \\
\text { pump } \\
\end{array}$ \\
\hline 29 & 4 & $\begin{array}{c}\text { Soon } \\
\text { afterwards }\end{array}$ & $\begin{array}{l}\text { C44.2 } \\
\text { C41.1 }\end{array}$ & $\begin{array}{l}0 \\
0 \\
\end{array}$ & $\begin{array}{l}\text { (S.28) } \\
\text { (S.28) }\end{array}$ \\
\hline $\begin{array}{l}\text { Sentence } \\
\text { number }\end{array}$ & $\begin{array}{c}\text { No. of } \\
\text { ties }\end{array}$ & Cohesive item & Type & Distance & Presupposed item \\
\hline & & $\begin{array}{c}\text { the } \\
\text { disease } \\
\text { slowed down } \\
\end{array}$ & $\begin{array}{c}\text { R23.6 } \\
\text { L1.6 } \\
\text { L5 } \\
\end{array}$ & $\begin{array}{c}\text { N.9 } \\
\text { N.9 } \\
\text { N.13 } \\
\end{array}$ & $\begin{array}{l}\text { the disease } \\
\text { the disease } \\
\text { spread quickly }\end{array}$ \\
\hline 30 & 5 & $\begin{array}{c}\text { He } \\
\text { cholera } \\
\text { spread } \\
\text { germs } \\
\text { gas }\end{array}$ & $\begin{array}{l}\text { R11.6 } \\
\text { L2.6 } \\
\text { L1.6 } \\
\text { L5 } \\
\text { L1.6 }\end{array}$ & $\begin{array}{c}\text { M.2 } \\
0 \\
\text { N.13 } \\
\text { N.2 } \\
\text { N.20 }\end{array}$ & $\begin{array}{c}\mathrm{He} \rightarrow \text { John Snow } \\
\text { disease } \\
\text { spread } \\
\text { polluted/dirty } \\
\text { gas } \\
\end{array}$ \\
\hline 31 & 10 & $\begin{array}{l}\text { another } \\
\text { part } \\
\text { London } \\
\text { he } \\
\text { found } \\
\text { evidence } \\
\text { two other } \\
\text { deaths } \\
\text { Broad Street } \\
\text { outbreak }\end{array}$ & $\begin{array}{c}\text { R33 } \\
\text { L3.8 } \\
\text { L1.6 } \\
\text { R11.6 } \\
\text { L1.6 } \\
\text { L1.6 } \\
\text { R33.9 } \\
\text { L1.8 } \\
\text { L1.6 } \\
\text { L1.6 }\end{array}$ & $\begin{array}{l}\text { N.2 } \\
\text { N.2 } \\
\text { N.3 } \\
\text { M.3 } \\
\text { N.3 } \\
\text { N.17 } \\
\text { N.10 } \\
\text { N.10 } \\
\text { N.2 } \\
\text { N.14 }\end{array}$ & $\begin{array}{c}\text { Broad Street } \\
\text { Broad Street } \\
\text { London } \\
\mathrm{He} \rightarrow \text { John Snow } \\
\text { found } \\
\text { evidence } \\
\text { Many of the deaths } \\
\text { deaths } \\
\text { Broad Street } \\
\text { outbreak }\end{array}$ \\
\hline 32 & 5 & $\begin{array}{c}\text { Broad Street } \\
\text { the } \\
\text { water/it } \\
\text { pump } \\
\text { house } \\
\end{array}$ & $\begin{array}{c}\text { L1.6 } \\
\text { R23.6 } \\
\text { L1.6 } \\
\text { L1.6 } \\
\text { L1.9 }\end{array}$ & $\begin{array}{c}0 \\
\text { N.7 } \\
\text { N.7 } \\
\text { N.3 } \\
\text { N.10 }\end{array}$ & $\begin{array}{l}\text { Broad Street } \\
\text { the water from the pump } \\
\text { the water from the pump } \\
\text { pump } \\
\text { houses }\end{array}$ \\
\hline 33 & 6 & $\begin{array}{c}\text { she/her } \\
\text { died } \\
\text { cholera } \\
\text { drinking } \\
\text { the } \\
\text { water }\end{array}$ & $\begin{array}{l}\text { R12.6 } \\
\text { L5 } \\
\text { L1.6 } \\
\text { L1.6 } \\
\text { R23.6 } \\
\text { L1.6 }\end{array}$ & $\begin{array}{c}0 \\
\text { N.1 } \\
\text { N.2 } \\
\text { N.8 } \\
0 \\
0\end{array}$ & $\begin{array}{c}\text { a woman } \\
\text { deaths } \\
\text { cholera } \\
\text { drunk } \\
\text { the water } \\
\text { the water }\end{array}$ \\
\hline 34 & 6 & $\begin{array}{c}\text { this } \\
\text { evidence } \\
\text { John Snow } \\
\text { polluted } \\
\text { water }\end{array}$ & $\begin{array}{l}\text { R21.6 } \\
\text { L1.6 } \\
\text { L1.6 } \\
\text { L1.6 } \\
\text { L1.6 }\end{array}$ & $\begin{array}{c}0 \\
\text { N.2 } \\
\text { N.7 } \\
\text { N.6 } \\
0\end{array}$ & $\begin{array}{c}\text { (S.33) } \\
\text { evidence } \\
\text { John Snow } \\
\text { polluted } \\
\text { water }\end{array}$ \\
\hline
\end{tabular}




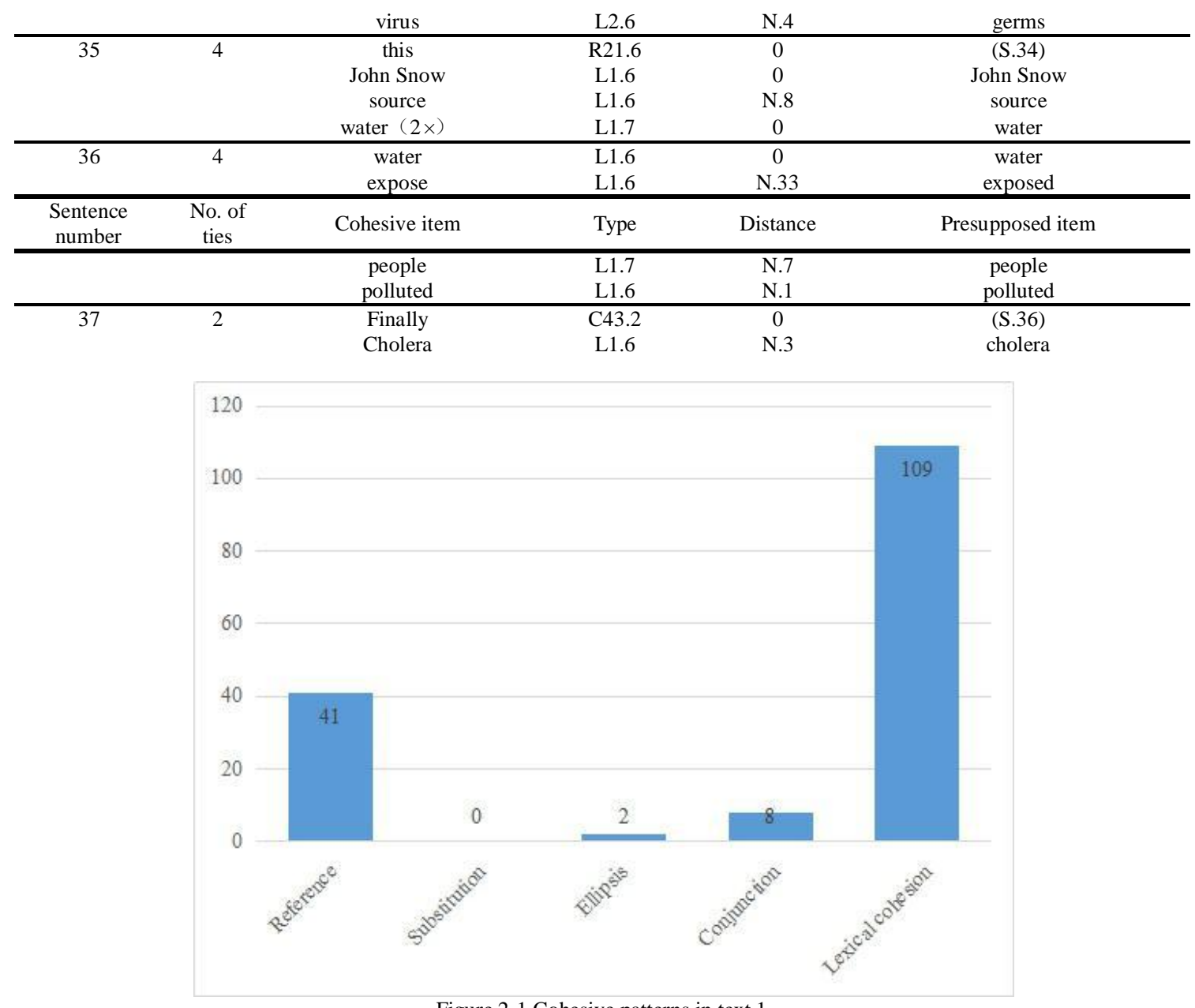

As it can be seen from the above histogram, among the five lexical cohesive devices, lexical cohesion appears 109 times which proved to be the most in the texts while reference secures the second place with 41 occurrences, but far less than the first one. Conjunction and ellipsis are even less, 8 and 2 occurrences respectively. Substitution accounts for nothing without occurring.

TABLE 2-2

The ANALYSIS OF COHESION IN TEXT 2

\begin{tabular}{|c|c|c|c|c|c|}
\hline $\begin{array}{l}\text { Sentence } \\
\text { number }\end{array}$ & $\begin{array}{c}\text { No. of } \\
\text { ties }\end{array}$ & Cohesion item & Type & Distance & Presupposed item \\
\hline \multirow[t]{2}{*}{2} & 2 & he & R11.6 & 0 & Nicolaus Copernicus \\
\hline & & them & $\mathrm{R} 14.6$ & 0 & (S.1) \\
\hline 3 & 1 & the sun & L5 & 0 & solar \\
\hline \multirow[t]{6}{*}{4} & 6 & Yet & $\mathrm{C} 21.1$ & 0 & $(\mathrm{~S} .3)$ \\
\hline & & he/him & R11.6 & M.1+N.1 & $\rightarrow$ he $\rightarrow$ Nicolaus Copernicus \\
\hline & & his & $\mathrm{R} 11.8$ & M.1+N.1 & $\rightarrow$ he $\rightarrow$ Nicolaus Copernicus \\
\hline & & theory & L4.6 & 0 & $\begin{array}{l}\text { that the earth was not ... system(S.2) } \\
\text { (S.3) }\end{array}$ \\
\hline & & such & R32.9 & 0 & that the earth was not ... system(S.2) \\
\hline & & an idea & L4.6 & 0 & that the earth was not ... system(S.2) \\
\hline \multirow[t]{4}{*}{5} & 4 & They & R14.6 & 0 & Christian Church \\
\hline & & God & L5 & 0 & Christian Church \\
\hline & & the earth & L1.6 & N.2 & the earth \\
\hline & & $\begin{array}{l}\text { the centre of the solar } \\
\text { system }\end{array}$ & L1.6 & N.2 & the earth \\
\hline \multirow[t]{3}{*}{6} & 3 & planets & L1.9 & N.2 & planets \\
\hline & & in the sky & L1.6 & N.2 & in the sky \\
\hline & & move & L5 & N.2 & movements \\
\hline 7 & 1 & Others & R33.6 & 0 & some planets \\
\hline \multirow[t]{3}{*}{8} & 4 & This & $\mathrm{R} 21.6$ & 0 & $\begin{array}{c}\text { some planets in the sky...loop(S.6) } \\
\text { (S.7) }\end{array}$ \\
\hline & & the earth & L1.6 & N.2 & the earth \\
\hline & & the centre of the & L1.6 & N.2 & the centre of the solar system \\
\hline
\end{tabular}




\begin{tabular}{|c|c|c|c|c|c|}
\hline & & $\begin{array}{l}\text { solar system } \\
\text { planets }\end{array}$ & $\begin{array}{l}\text { L1.6 } \\
\text { L1.7 }\end{array}$ & $\begin{array}{l}\text { N.2 } \\
\text { N.1 }\end{array}$ & $\begin{array}{c}\text { solar system } \\
\text { planets }\end{array}$ \\
\hline $\begin{array}{l}\text { Sentence } \\
\text { number }\end{array}$ & $\begin{array}{c}\text { No. of } \\
\text { ties }\end{array}$ & Cohesion item & Type & Distance & Presupposed item \\
\hline \multirow[t]{2}{*}{9} & 3 & $\begin{array}{l}\text { Copernicus } \\
\text { these }\end{array}$ & $\begin{array}{c}\text { L1.6 } \\
\text { R21.6 }\end{array}$ & $\begin{array}{l}\text { N.7 } \\
\text { N.1 }\end{array}$ & $\begin{array}{c}\text { Nicolaus Copernicus } \\
\text { some planets in the sky...loop(S.6) } \\
(\text { S.7) }\end{array}$ \\
\hline & & problems & L1.6 & N.2 & The problem \\
\hline 10 & 4 & $\begin{array}{c}\mathrm{He} \\
\text { observations } \\
\text { the stars } \\
\text { them }\end{array}$ & $\begin{array}{l}\text { R11.6 } \\
\text { L5 } \\
\text { L2.6 } \\
\text { R14.6 } \\
\end{array}$ & $\begin{array}{c}0 \\
\text { N.3 } \\
\text { N.1 } \\
0 \\
\end{array}$ & $\begin{array}{c}\text { Copernicus } \\
\text { noticed } \\
\text { planets } \\
\text { these problems }\end{array}$ \\
\hline 11 & 4 & $\begin{array}{l}\text { But } \\
\text { his } \\
\text { theory } \\
\text { do that }\end{array}$ & $\begin{array}{l}\mathrm{C} 21.2 \\
\text { R11.8 } \\
\text { L1.6 } \\
\text { S24 }\end{array}$ & $\begin{array}{c}0 \\
\text { M.1 } \\
\text { N.6 } \\
0\end{array}$ & $\begin{array}{c}\mathrm{He} \rightarrow \text { Copernicus } \\
\text { theory } \\
(\mathrm{S} .10)\end{array}$ \\
\hline 12 & 4 & $\begin{array}{c}\text { So } \\
\text { he }(2 \times) \\
\text { it }(2 \times) \\
\text { theory }\end{array}$ & $\begin{array}{l}\text { C31.1 } \\
\text { R11.6 } \\
\text { R13.6 } \\
\text { L1.6 }\end{array}$ & $\begin{array}{c}0 \\
\text { M.2 } \\
0 \\
0\end{array}$ & $\begin{array}{c}\text { his } \rightarrow \mathrm{He} \rightarrow \text { Copernicus } \\
\text { theory } \\
\text { theory }\end{array}$ \\
\hline 13 & 3 & $\begin{array}{c}\text { In } 1514 \\
\text { he } \\
\text { it }\end{array}$ & $\begin{array}{c}\text { L5 } \\
\text { R11.6 } \\
\text { R13.6 } \\
\end{array}$ & $\begin{array}{c}0 \\
\text { M.3 } \\
0 \\
\end{array}$ & $\begin{array}{c}\text { between } 1510 \text { and } 1514 \\
\rightarrow \text { he } \rightarrow \text { his } \rightarrow \text { He } \rightarrow \text { Copernicus } \\
\text { theory }\end{array}$ \\
\hline 14 & 3 & $\begin{array}{l}\text { he } \\
\text { old } \\
\text { theory }\end{array}$ & $\begin{array}{l}\text { R11.6 } \\
\text { L5 } \\
\text { L1.8 } \\
\end{array}$ & $\begin{array}{l}\text { M.4 } \\
\text { N.2 } \\
\text { N.1 }\end{array}$ & $\begin{array}{c}\rightarrow \text { he } \rightarrow \text { he } \rightarrow \text { his } \rightarrow \text { He } \rightarrow \text { Copernicus } \\
\text { new } \\
\text { theory }\end{array}$ \\
\hline 15 & 6 & $\begin{array}{c}\text { he } \\
\text { sun } \\
\text { the centre of the solar } \\
\text { system }\end{array}$ & $\begin{array}{l}\text { R11.6 } \\
\text { L1.6 } \\
\text { L1.6 }\end{array}$ & $\begin{array}{l}\text { M.5 } \\
\text { N.11 } \\
\text { N.6 }\end{array}$ & $\begin{array}{l}\qquad \rightarrow \text { he } \rightarrow \ldots \rightarrow \text { Copernicus } \\
\text { the sun } \\
\text { the centre of the solar system }\end{array}$ \\
\hline \multirow[t]{2}{*}{$\begin{array}{l}\text { Sentence } \\
\text { number }\end{array}$} & $\begin{array}{l}\text { No. of } \\
\text { ties }\end{array}$ & Cohesion item & Type & Distance & Presupposed item \\
\hline & & $\begin{array}{l}\text { the planets } \\
\text { going round }(2 \times) \\
\text { the earth }\end{array}$ & $\begin{array}{l}\text { L1.9 } \\
\text { L5 } \\
\text { L1.6 } \\
\end{array}$ & $\begin{array}{l}\text { N.6 } \\
\text { N.6 } \\
\text { N.6 } \\
\end{array}$ & $\begin{array}{c}\text { planets } \\
\text { went round } \\
\text { the earth } \\
\end{array}$ \\
\hline 16 & 11 & $\begin{array}{c}\mathrm{He} \\
\text { also } \\
\text { suggested/explained } \\
\text { the earth } \\
\text { went round } \\
\text { the sun } \\
\text { changes } \\
\text { movement } \\
\text { the planets } \\
\text { brightness } \\
\text { the stars }\end{array}$ & $\begin{array}{l}\text { R11.6 } \\
\text { C11.1 } \\
\text { L5 } \\
\text { L1.6 } \\
\text { L5 } \\
\text { L1.6 } \\
\text { L1.9 } \\
\text { L5 } \\
\text { L1.6 } \\
\text { L5 } \\
\text { L2.6 }\end{array}$ & $\begin{array}{c}\text { M.6 } \\
0 \\
0 \\
0 \\
0 \\
0 \\
\text { N.14 } \\
\text { N.9 } \\
0 \\
\text { N.8 } \\
0 \\
\end{array}$ & $\begin{array}{c}\rightarrow \text { he } \rightarrow \text {... } \rightarrow \text { Copernicus } \\
\text { (S.15) } \\
\text { showed } \\
\text { the earth } \\
\text { going round } \\
\text { sun } \\
\text { changes } \\
\text { move } \\
\text { the planets } \\
\text { brighter } \\
\text { the planets }\end{array}$ \\
\hline 17 & 5 & $\begin{array}{c}\text { His }(2 \times) \\
\text { friends } \\
\text { him } \\
\text { ideas } \\
\text { Copernicus }\end{array}$ & $\begin{array}{l}\text { R11.8 } \\
\text { L1.6 } \\
\text { R11.6 } \\
\text { L1.7 } \\
\text { L1.6 }\end{array}$ & $\begin{array}{l}\text { M.7 } \\
\text { N.3 } \\
\text { M.7 } \\
\text { N.12 } \\
\text { N.7 }\end{array}$ & $\begin{aligned} \rightarrow & \rightarrow \mathrm{He} \rightarrow \ldots \rightarrow \text { Copernicus } \\
& \text { friends } \\
\rightarrow \mathrm{He} & \rightarrow \ldots \rightarrow \text { Copernicus } \\
& \text { idea } \\
& \text { Copernicus }\end{aligned}$ \\
\hline 18 & 5 & $\begin{array}{c}\operatorname{He}(3 \times) \\
\text { the Christian Church } \\
\text { published } \\
\text { it } \\
\text { in1543 }\end{array}$ & $\begin{array}{l}\text { R11.6 } \\
\text { L1.6 } \\
\text { L1.6 } \\
\text { L13.6 } \\
\text { L5 }\end{array}$ & $\begin{array}{c}0 \\
\text { N.13 } \\
0 \\
0 \\
\text { N.4 }\end{array}$ & $\begin{array}{c}\text { Copernicus } \\
\text { the Christian Church } \\
\text { publish } \\
\text { ideas } \\
\text { in } 1514\end{array}$ \\
\hline 19 & 2 & he & $\mathrm{R} 11.6$ & M.1 & $\rightarrow \mathrm{He} \rightarrow$ Copernicus \\
\hline \multirow[t]{2}{*}{$\begin{array}{l}\text { Sentence } \\
\text { number }\end{array}$} & $\begin{array}{c}\text { No. of } \\
\text { ties }\end{array}$ & Cohesion item & Type & Distance & Presupposed item \\
\hline & & careful & $\mathrm{L} 2.6$ & N.1 & cautious \\
\hline 20 & 6 & $\begin{array}{c}\text { The Christian Church } \\
\text { his } \\
\text { theory } \\
\text { God's } \\
\text { idea } \\
\text { attacked } \\
\end{array}$ & $\begin{array}{l}\text { L1.6 } \\
\text { R11.8 } \\
\text { L2.6 } \\
\text { L1.6 } \\
\text { L1.8 } \\
\text { L1.6 } \\
\end{array}$ & $\begin{array}{c}0 \\
\text { M.1 } \\
\text { N.1 } \\
\text { N.13 } \\
\text { N.17 } \\
\text { N.1 } \\
\end{array}$ & $\begin{array}{c}\text { the Christian Church } \\
\rightarrow \mathrm{He} \rightarrow \text { Copernicus } \\
\text { ideas } \\
\text { God } \\
\text { ideas } \\
\text { attacked } \\
\end{array}$ \\
\hline 21 & 6 & $\begin{array}{c}\text { Yet } \\
\text { Copernicus' } \\
\text { theory } \\
\text { now } \\
\text { ideas } \\
\text { the universe }\end{array}$ & $\begin{array}{l}\text { C21.1 } \\
\text { L1.6 } \\
\text { L1.6 } \\
\text { L5 } \\
\text { L1.9 } \\
\text { L2.6 }\end{array}$ & $\begin{array}{c}0 \\
\mathrm{~N} .3 \\
0 \\
\mathrm{~N} .2 \\
0 \\
\mathrm{~N} .5\end{array}$ & $\begin{array}{c}\text { (S.20) } \\
\text { Copernicus } \\
\text { theory } \\
\text { in } 1543 \\
\text { idea } \\
\text { the solar system }\end{array}$ \\
\hline 22 & 9 & His & $\mathrm{R} 11.8$ & 0 & Copernicus' \\
\hline
\end{tabular}




\begin{tabular}{|c|c|c|c|c|c|}
\hline & & $\begin{array}{c}\text { theory } \\
\text { the Christian } \\
\text { idea } \\
\text { earth }(2 \times) \\
\text { God } \\
\text { created } \\
\text { the centre of } \\
\text { the universe }\end{array}$ & $\begin{array}{l}\text { L1.6 } \\
\text { L1.6 } \\
\text { L1.9 } \\
\text { L1.6 } \\
\text { L1.6 } \\
\text { L2.6 } \\
\text { L1.6 } \\
\text { L1.6 }\end{array}$ & $\begin{array}{c}0 \\
\text { N.1 } \\
0 \\
\text { N.5 } \\
\text { N.1 } \\
\text { N.6 } \\
\text { N.6 } \\
0 \\
\end{array}$ & $\begin{array}{l}\text { theory } \\
\text { the Christian } \\
\text { ideas } \\
\text { the earth } \\
\text { God's } \\
\text { made } \\
\text { the centre of } \\
\text { the universe }\end{array}$ \\
\hline 23 & 3 & Copernicus & L1.6 & N.1 & Copernicus' \\
\hline \multirow[t]{2}{*}{$\begin{array}{c}\text { Sentence } \\
\text { number }\end{array}$} & $\begin{array}{c}\text { No. of } \\
\text { ties }\end{array}$ & Cohesion item & Type & Distance & Presupposed item \\
\hline & & $\begin{array}{c}\text { showed } \\
\text { this }\end{array}$ & $\begin{array}{c}\text { L5 } \\
\text { R21.6 }\end{array}$ & $\begin{array}{c}\text { N.6 } \\
0\end{array}$ & $\begin{array}{l}\text { suggested/explained } \\
\text { the Christian idea of gravity, } \\
\text { which...universe.(S.22) }\end{array}$ \\
\hline 24 & 4 & $\begin{array}{l}\text { Now } \\
\text { people } \\
\text { his } \\
\text { theory }\end{array}$ & $\begin{array}{l}\text { L5 } \\
\text { L1.6 } \\
\text { R11.8 } \\
\text { L1.6 }\end{array}$ & $\begin{array}{l}\text { N.2 } \\
\text { N.4 } \\
0 \\
\text { N.1 }\end{array}$ & $\begin{array}{c}\text { now } \\
\text { people } \\
\text { Copernicus } \\
\text { theory }\end{array}$ \\
\hline
\end{tabular}

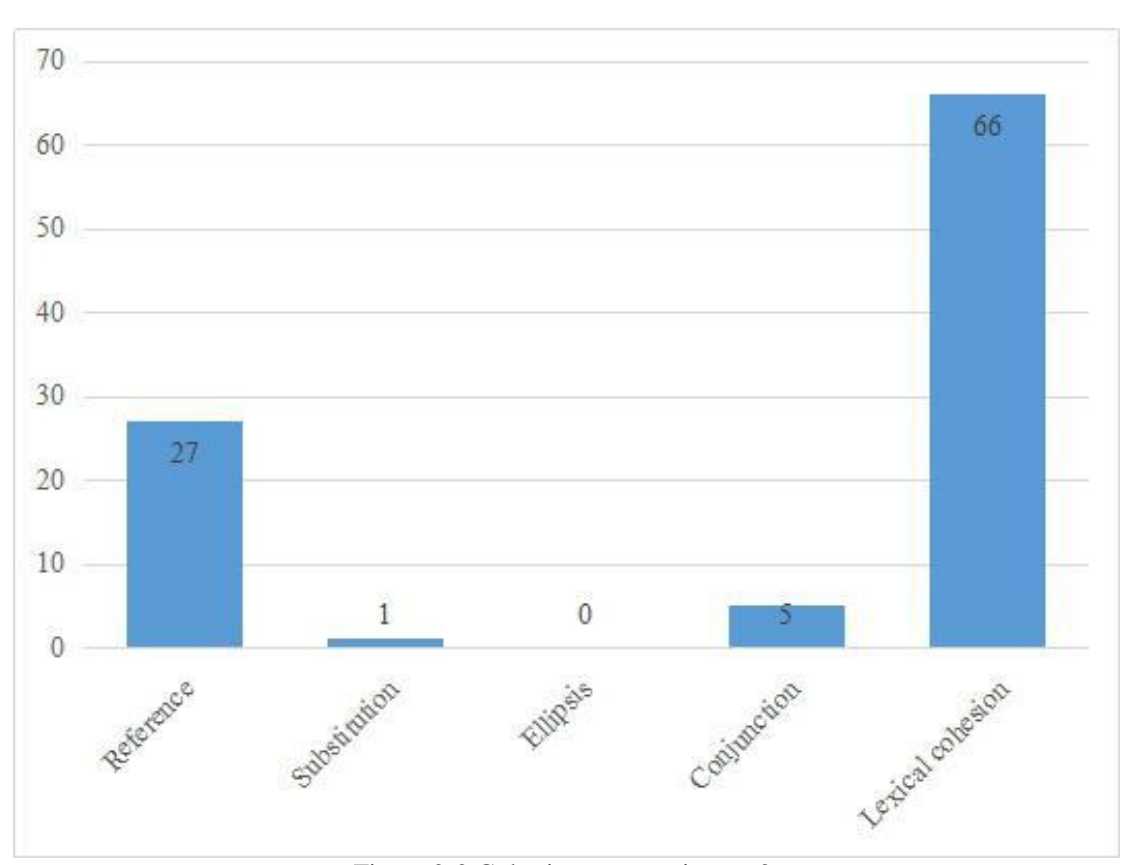

Figure 2-2 Cohesive patterns in text 2

As it can be seen from the above histogram, among the five lexical cohesive devices, lexical cohesion appears 66 times which proved to be the most in the texts while reference secures the second place with 27 occurrences, but far less than the first one. Conjunction and substitution are even less, 5 and 1 occurrences respectively. Ellipsis accounts for nothing without occurring.

TABLE 2-3

THE ANALYSIS OF COHESION IN TEXT 3

\begin{tabular}{|c|c|c|c|c|c|}
\hline $\begin{array}{l}\text { Sentence } \\
\text { number }\end{array}$ & $\begin{array}{c}\text { No. of } \\
\text { ties }\end{array}$ & Cohesion item & Type & Distance & Presupposed item \\
\hline \multirow[t]{3}{*}{2} & 2 & this & R21.6 & 0 & why...Ireland.(S.1) \\
\hline & & question & L4.6 & 0 & why...Ireland.(S.1) \\
\hline & & British & L5 & 0 & England...Northern Ireland \\
\hline \multirow[t]{2}{*}{3} & 2 & First & $\mathrm{C} 43.1$ & 0 & $(\mathrm{~S} .2)$ \\
\hline & & England & L1.6 & N.1 & England \\
\hline \multirow[t]{2}{*}{4} & 2 & Wales & $\mathrm{L} 1.6$ & N.2 & Wales \\
\hline & & it & $\mathrm{R} 13.6$ & 0 & England \\
\hline \multirow[t]{4}{*}{5} & 4 & Now & L5 & 0 & in the thirteen century \\
\hline & & people & L1.6 & N.3 & people \\
\hline & & England & L1.6 & N.1 & England \\
\hline & & Wales & L1.6 & 0 & Wales \\
\hline \multirow[t]{4}{*}{6} & 5 & Next & $\mathrm{C} 41.1$ & 0 & (S.2) \\
\hline & & England & L1.6 & N.1 & England \\
\hline & & Wales & L1.6 & 0 & Wales \\
\hline & & Scotland & L1.6 & N.4 & Scotland \\
\hline
\end{tabular}




\begin{tabular}{|c|c|c|c|c|c|}
\hline & & in the seventeen century & L5 & 0 & Now \\
\hline \multirow[t]{4}{*}{7} & 4 & this & $\mathrm{R} 21.6$ & 0 & (S.6) \\
\hline & & Scotland & $\mathrm{L} 1.6$ & 0 & Scotland \\
\hline & & England & L1.6 & N.1 & England \\
\hline & & Wales & L1.6 & 0 & Wales \\
\hline 8 & 9 & Finally & $\mathrm{C} 43.2$ & 0 & (S.7) \\
\hline \multirow[t]{8}{*}{$\begin{array}{l}\text { Sentence } \\
\text { number }\end{array}$} & $\begin{array}{c}\text { No. of } \\
\text { ties }\end{array}$ & Cohesion item & Type & Distance & Presupposed item \\
\hline & & English & L5 & 0 & England \\
\hline & & $\begin{array}{c}\text { in the early twentieth } \\
\text { century }\end{array}$ & L5 & 0 & in the seventeen century \\
\hline & & the United Kingdom & L5 & N.1 & "Great Britain" \\
\hline & & Ireland & $\mathrm{L} 1.7$ & N.6 & Ireland \\
\hline & & connected & L5 & N.2 & included \\
\hline & & in the same (peaceful) way & C15.1 & 0 & (S.7) \\
\hline & & peaceful & L5 & 0 & conflict \\
\hline \multirow[t]{3}{*}{9} & 3 & However & $\mathrm{C} 21.3$ & 0 & (S.8) \\
\hline & & Ireland & $\mathrm{L} 1.8$ & 0 & Ireland \\
\hline & & government & $\mathrm{L} 1.8$ & 0 & government \\
\hline \multirow[t]{8}{*}{10} & 8 & So & C31.1 & 0 & (S.9) \\
\hline & & Northern & L5 & 0 & southern \\
\hline & & Ireland & $\mathrm{L} 1.8$ & 0 & Ireland \\
\hline & & joined & L5 & 0 & connected \\
\hline & & England & L1.6 & N.2 & England \\
\hline & & Wales & L1. 6 & N.2 & Wales \\
\hline & & Scotland & L1.6 & N.2 & Scotland \\
\hline & & the United Kingdom & L1.6 & N.1 & the United Kingdom \\
\hline 11 & 2 & $\begin{array}{c}\text { the } \\
\text { countries } \\
\end{array}$ & R23.6 & 0 & $\begin{array}{l}\text { the Northern Ireland, England, Wales, Scotland } \\
\text { the Northern Ireland, England, Wales, Scotland }\end{array}$ \\
\hline \multirow[t]{2}{*}{12} & 5 & Northern Ireland & L5 & 0 & four countries \\
\hline & & England & L5 & 0 & four countries \\
\hline \multirow[t]{4}{*}{$\begin{array}{l}\text { Sentence } \\
\text { number }\end{array}$} & $\begin{array}{l}\text { No. of } \\
\text { ties }\end{array}$ & Cohesion item & Type & Distance & Presupposed item \\
\hline & & Scotland & L5 & 0 & four countries \\
\hline & & $\operatorname{different}(2 \times)$ & L1.6 & 0 & different \\
\hline & & systems & L5 & 0 & institutions \\
\hline \multirow[t]{2}{*}{13} & 2 & England & L1.6 & 0 & England \\
\hline & & the four countries & L1.6 & N.1 & the four countries \\
\hline \multirow[t]{5}{*}{14} & 5 & The $(2 \times)$ & $\mathrm{R} 23.6$ & 0 & three zones \\
\hline & & zone $(2 \times)$ & L1.7 & 0 & three zones \\
\hline & & called & L1.9 & N.4 & called \\
\hline & & England & L1. 6 & 0 & England \\
\hline & & Scotland & L1.6 & N.1 & Scotland \\
\hline \multirow[t]{5}{*}{15} & 5 & find & L1.6 & N.9 & find \\
\hline & & the south & L1.6 & 0 & the South \\
\hline & & the Midlands & L1.6 & 0 & the Midlands \\
\hline & & the North & L1.6 & 0 & the North \\
\hline & & England & L1.6 & 0 & England \\
\hline \multirow[t]{4}{*}{16} & 4 & these & R21.6 & 0 & the industrial cities \\
\hline & & cities & L1.6 & 0 & cities \\
\hline & & football teams & L1.6 & N.3 & football teams \\
\hline & & two & $\mathrm{L} 12.2$ & N.3 & football teams \\
\hline 17 & 1 & the industrial cities & L1.6 & N.1 & the industrial cities \\
\hline 18 & 1 & towns & L5 & 0 & cities \\
\hline \multirow[t]{4}{*}{19} & 4 & There & $\mathrm{R} 22.7$ & 0 & older but smaller towns \\
\hline & & find out & L1.6 & N.3 & find \\
\hline & & British & L5 & N.3 & England \\
\hline & & history & L5 & 0 & historical \\
\hline 20 & 2 & historical & L5 & 0 & history \\
\hline \multirow[t]{2}{*}{$\begin{array}{l}\text { Sentence } \\
\text { number }\end{array}$} & $\begin{array}{l}\text { No. of } \\
\text { ties }\end{array}$ & Cohesion item & Type & Distance & Presupposed item \\
\hline & & London & L5 & 0 & British \\
\hline \multirow[t]{3}{*}{21} & 3 & It & R13.6 & 0 & London \\
\hline & & national & L5 & N.1 & British \\
\hline & & government & L1.9 & N.11 & government \\
\hline 22 & 8 & It & R13.6 & M.1 & London \\
\hline & & $\operatorname{oldest}(3 \times)$ & L5 & N.3 & older \\
\hline & & built & L1.6 & N.3 & built \\
\hline & & Romans & L1.6 & N.3 & Romans \\
\hline & & building & L1.6 & N.1 & buildings \\
\hline & & begun & $\mathrm{L} 2.6$ & N.3 & built \\
\hline
\end{tabular}




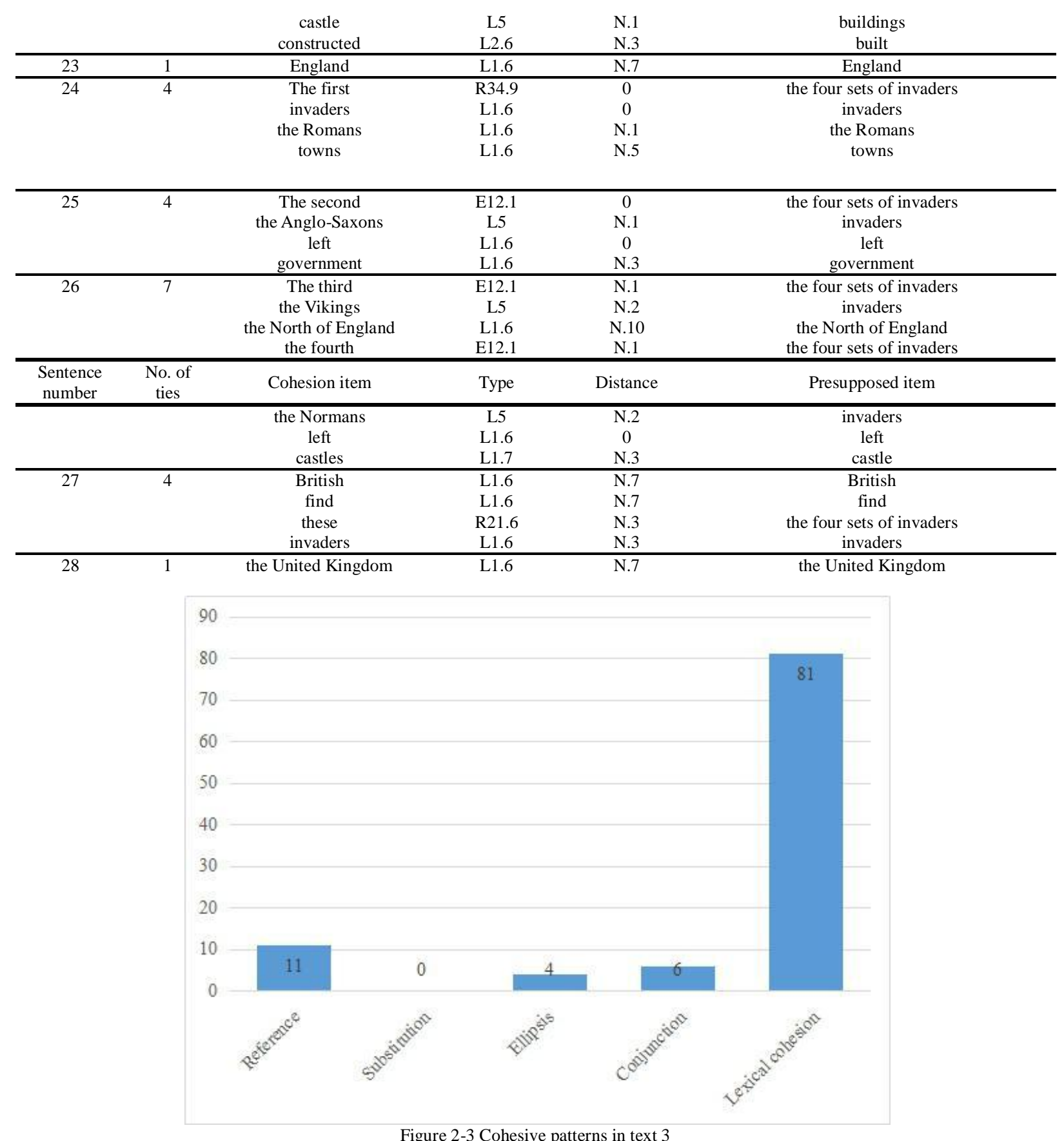

As it can be seen from the above histogram, among the five lexical cohesive devices, lexical cohesion appears 81 times which proved to be the most in the texts while reference, conjunction and ellipsis are much less, 11,6 and 4 occurrences respectively. Substitution accounts for nothing without occurring.

TABLE 2-4

THE ANALYSIS OF COHESION IN TEXT 4

\begin{tabular}{|c|c|c|c|c|c|}
\hline $\begin{array}{l}\text { Sentence } \\
\text { number }\end{array}$ & $\begin{array}{l}\text { No. of } \\
\text { ties }\end{array}$ & Cohesion item & Type & Distance & Presupposed item \\
\hline \multirow[t]{3}{*}{2} & 3 & That & R22.6 & 0 & $(\mathrm{~S} .1)$ \\
\hline & & much & $\mathrm{R} 34.7$ & 0 & one degree \\
\hline & & it & $\mathrm{R} 13.6$ & 0 & (S.1) \\
\hline \multirow[t]{3}{*}{3} & 3 & So & C31.1 & 0 & $(\mathrm{~S} .2)$ \\
\hline & & this & $\mathrm{R} 21.6$ & N.1 & (S.1) \\
\hline & & it & R13.6 & N.1 & (S.1) \\
\hline \multirow[t]{2}{*}{4} & 2 & these & $\mathrm{R} 21.6$ & 0 & (S.3) \\
\hline & & questions & L4.6 & 0 & (S.3) \\
\hline \multirow[t]{2}{*}{5} & 5 & the earth & L1.6 & N.3 & the earth \\
\hline & & warmer/warming & L5 & N.3 & the temperature...rose \\
\hline
\end{tabular}




\begin{tabular}{|c|c|c|c|c|c|}
\hline & & $\begin{array}{l}\text { this } \\
\text { global } \\
\text { natural }\end{array}$ & $\begin{array}{l}\text { R21.6 } \\
\text { L5 } \\
\text { L1.6 }\end{array}$ & $\begin{array}{l}\text { N.3 } \\
\text { N.3 } \\
\text { N.2 }\end{array}$ & $\begin{array}{c}\text { (S.1) } \\
\text { the earth } \\
\text { natural }\end{array}$ \\
\hline 6 & 6 & $\begin{array}{c}\text { the } \\
\text { increase } \\
\text { the earth } \\
\text { the temperature } \\
\text { burning } \\
\text { natural } \\
\end{array}$ & $\begin{array}{c}\text { R23.6 } \\
\text { L1.6 } \\
\text { L1.6 } \\
\text { L1.6 } \\
\text { L5 } \\
\text { L1.6 }\end{array}$ & $\begin{array}{c}\text { N.3 } \\
\text { N.3 } \\
0 \\
\text { N.4 } \\
0 \\
0 \\
\end{array}$ & $\begin{array}{c}\text { a rapid increase } \\
\text { increase } \\
\text { the earth } \\
\text { the temperature } \\
\text { human activity } \\
\text { natural }\end{array}$ \\
\hline 7 & 2 & $\begin{array}{c}\text { this } \\
\text { process } \\
\end{array}$ & $\begin{array}{l}\text { R21.6 } \\
\text { L4.6 }\end{array}$ & $\begin{array}{l}0 \\
0 \\
\end{array}$ & $\begin{array}{l}\text { the burning...to produce energy } \\
\text { the burning...to produce energy }\end{array}$ \\
\hline 8 & 4 & $\begin{array}{l}\text { Dr Janice Foster } \\
\text { a natural phenomenon }\end{array}$ & $\begin{array}{l}\text { L5 } \\
\text { L1.6 }\end{array}$ & $\begin{array}{l}\text { N.1 } \\
\text { N.2 }\end{array}$ & $\begin{array}{c}\text { All scientists } \\
\text { a (random but) natural phenomenon }\end{array}$ \\
\hline
\end{tabular}

\begin{tabular}{|c|c|c|c|c|c|}
\hline $\begin{array}{l}\text { Sentence } \\
\text { number }\end{array}$ & $\begin{array}{c}\text { No. of } \\
\text { ties }\end{array}$ & Cohesion item & Type & Distance & Presupposed item \\
\hline & & $\begin{array}{c}\text { scientists } \\
\text { 'greenhouse' }\end{array}$ & $\begin{array}{l}\text { L1.6 } \\
\text { L1.6 } \\
\end{array}$ & $\begin{array}{c}\mathrm{N} .1 \\
0\end{array}$ & $\begin{array}{c}\text { scientists } \\
\text { 'greenhouse' }\end{array}$ \\
\hline 9 & 5 & $\begin{array}{c}\text { This } \\
\text { gases } \\
\text { warm } \\
\text { the earth } \\
\text { carbon dioxide }\end{array}$ & $\begin{array}{l}\text { R21.6 } \\
\text { L1.6 } \\
\text { L1.6 } \\
\text { L1.6 } \\
\text { L1.6 }\end{array}$ & $\begin{array}{l}0 \\
\text { N.1 } \\
\text { N.3 } \\
\text { N.2 } \\
\text { N.1 }\end{array}$ & $\begin{array}{l}\text { the'green house effect' } \\
\text { gases } \\
\text { warming } \\
\text { the earth } \\
\text { carbon dioxide }\end{array}$ \\
\hline 10 & 3 & $\begin{array}{c}\text { the 'green house effect' } \\
\text { the earth } \\
\text { degrees Celsius }\end{array}$ & $\begin{array}{l}\text { L1.6 } \\
\text { L1.6 } \\
\text { L2.6 } \\
\end{array}$ & $\begin{array}{c}\text { N.1 } \\
0 \\
\text { N.8 } \\
\end{array}$ & $\begin{array}{c}\text { the 'green house effect' } \\
\text { the earth } \\
\text { degree Fahrenheit }\end{array}$ \\
\hline 11 & 3 & $\begin{array}{c}\text { So } \\
\text { those } \\
\text { gases }\end{array}$ & $\begin{array}{l}\text { C31.1 } \\
\text { R22.6 } \\
\text { L1.6 }\end{array}$ & $\begin{array}{c}0 \\
\text { N.3 } \\
\text { N.3 }\end{array}$ & $\begin{array}{c}(\text { S.9) } \\
\text { "greenhouse" gases } \\
\text { "greenhouse" gases }\end{array}$ \\
\hline 12 & 4 & $\begin{array}{c}\text { quantities } \\
\text { extra } \\
\text { carbon dioxide } \\
\text { the atmosphere } \\
\end{array}$ & $\begin{array}{c}\text { L2.8 } \\
\text { R33.9 } \\
\text { L1.8 } \\
\text { L1.6 }\end{array}$ & $\begin{array}{l}\text { N.2 } \\
\text { N.2 } \\
\text { N.2 } \\
\text { N.2 }\end{array}$ & $\begin{array}{l}\text { amounts } \\
\text { carbon dioxide } \\
\text { carbon dioxide } \\
\text { the atmosphere }\end{array}$ \\
\hline 13 & 9 & $\begin{array}{c}\text { It } \\
\text { more } \\
\text { heat } \\
\text { trapped } \\
\text { the atmosphere } \\
\text { causing } \\
\text { global } \\
\text { temperature }\end{array}$ & $\begin{array}{c}\text { R13.6 } \\
\text { R33.9 } \\
\text { L1.8 } \\
\text { L1.6 } \\
\text { L1.6 } \\
\text { L1.6 } \\
\text { L5 } \\
\text { L1.6 }\end{array}$ & $\begin{array}{c}0 \\
0 \\
\text { N.3 } \\
\text { N.3 } \\
0 \\
\text { N.7 } \\
\text { N.2 } \\
\text { N.6 }\end{array}$ & $\begin{array}{c}\text { (S.12) } \\
\text { heat } \\
\text { heat } \\
\text { trap } \\
\text { the atmosphere } \\
\text { caused } \\
\text { the earth } \\
\text { temperature }\end{array}$ \\
\hline $\begin{array}{l}\text { Sentence } \\
\text { number }\end{array}$ & $\begin{array}{c}\text { No. of } \\
\text { ties }\end{array}$ & Cohesion item & Type & Distance & Presupposed item \\
\hline & & go up & L5 & N.6 & increase \\
\hline 14 & 2 & $\begin{array}{c}\text { carbon dioxide } \\
\text { increased }\end{array}$ & $\begin{array}{l}\mathrm{L} 1.6 \\
\mathrm{~L} 5\end{array}$ & $\begin{array}{c}\mathrm{N} .1 \\
0\end{array}$ & $\begin{array}{c}\text { carbon dioxide } \\
\text { go up } \\
\end{array}$ \\
\hline 15 & 5 & $\begin{array}{c}\text { scientist } \\
\text { amount } \\
\text { carbon dioxide } \\
\text { the atmosphere } \\
\text { from } 1957 \text { to } 1997\end{array}$ & $\begin{array}{l}\text { L1.7 } \\
\text { L2.9 } \\
\text { L1.6 } \\
\text { L1.6 } \\
\text { L5 }\end{array}$ & $\begin{array}{c}\mathrm{N} .8 \\
\mathrm{~N} .2 \\
0 \\
\mathrm{~N} .2 \\
0\end{array}$ & $\begin{array}{c}\text { scientists } \\
\text { quantities } \\
\text { carbon dioxide } \\
\text { the atmosphere } \\
\text { over the last } 100 \text { to } 150\end{array}$ \\
\hline 16 & 6 & $\begin{array}{c}\mathrm{He} \\
\text { these } \\
\text { years } \\
\text { carbon dioxide } \\
\text { the atmosphere } \\
\text { went up }\end{array}$ & $\begin{array}{l}\text { R11.6 } \\
\text { R21.6 } \\
\text { L5 } \\
\text { L1.6 } \\
\text { L1.6 } \\
\text { L5 }\end{array}$ & $\begin{array}{l}0 \\
0 \\
0 \\
0 \\
0 \\
0\end{array}$ & $\begin{array}{c}\text { Charles Keeling } \\
\text { from } 1957 \text { to } 1997 \\
\text { from } 1957 \text { to } 1997 \\
\text { carbon dioxide } \\
\text { the atmosphere } \\
\text { increased }\end{array}$ \\
\hline 17 & 4 & $\begin{array}{c}\text { scientists } \\
\text { accept } \\
\text { this } \\
\text { data }\end{array}$ & $\begin{array}{c}\text { L1.7 } \\
\text { L2.1 } \\
\text { R21.6 } \\
\text { L4.6 }\end{array}$ & $\begin{array}{c}\text { N.1 } \\
\text { N.10 } \\
0 \\
0 \\
\end{array}$ & $\begin{array}{c}\text { scientist } \\
\text { subscribe to } \\
\text { (S.16) } \\
\text { (S.16) }\end{array}$ \\
\hline 18 & 9 & $\begin{array}{l}\text { They } \\
\text { also } \\
\text { agree } \\
\text { burning } \\
\text { fossil fuels } \\
\text { resulted in } \\
\text { this } \\
\text { increase }\end{array}$ & $\begin{array}{c}\text { R14.6 } \\
\text { C11.1 } \\
\text { L2.1 } \\
\text { L1.6 } \\
\text { L1.6 } \\
\text { L2.1 } \\
\text { R21.6 } \\
\text { L5 }\end{array}$ & $\begin{array}{c}0 \\
0 \\
0 \\
\text { N.12 } \\
\text { N.12 } \\
\text { N.4 } \\
\text { N.1 } \\
\text { N.1 }\end{array}$ & $\begin{array}{l}\text { all scientist } \\
\text { (S.17) } \\
\text { accept } \\
\text { burning } \\
\text { fossil fuels } \\
\text { causing } \\
\text { (S.16) } \\
\text { went up }\end{array}$ \\
\hline $\begin{array}{l}\text { Sentence } \\
\text { number }\end{array}$ & $\begin{array}{l}\text { No. of } \\
\text { ties }\end{array}$ & Cohesion item & Type & Distance & Presupposed item \\
\hline & & carbon dioxide & L1.6 & N.1 & carbon dioxide \\
\hline
\end{tabular}




\begin{tabular}{|c|c|c|c|c|c|}
\hline & & $\begin{array}{l}\text { temperature } \\
\text { increase }\end{array}$ & $\begin{array}{l}\mathrm{L} 1.6 \\
\mathrm{~L} 1.6\end{array}$ & $\begin{array}{c}\text { N.5 } \\
0\end{array}$ & $\begin{array}{c}\text { temperature } \\
\text { increase } \\
\end{array}$ \\
\hline \multirow[t]{6}{*}{20} & 6 & Dr Janice Foster & L1.6 & N.11 & Dr Janice Foster \\
\hline & & over the next 100 & L5 & N.4 & from 1957 to 1997 \\
\hline & & years & $\mathrm{L} 1.9$ & N.3 & years \\
\hline & & amount & L1.6 & N.4 & amount \\
\hline & & warming & L1.6 & N.10 & warm \\
\hline & & degrees Celsius & L1.6 & N.9 & degrees Celsius \\
\hline \multirow[t]{4}{*}{21} & 4 & However & $\mathrm{C} 21.3$ & 0 & (S.20) \\
\hline & & scientists & L1.6 & N.3 & scientists \\
\hline & & this & $\mathrm{R} 21.6$ & 0 & (S.20) \\
\hline & & rise & $\mathrm{L} 2.6$ & 0 & the amount of warming \\
\hline \multirow[t]{4}{*}{22} & 4 & Dr Foster & L1.6 & N.1 & Dr Janice Foster \\
\hline & & temperature & $\mathrm{L} 1.6$ & N.2 & temperature \\
\hline & & increases & L5 & 0 & rise \\
\hline & & 5 degrees & L1.6 & 0 & 5 degrees \\
\hline 23 & 1 & She & $\mathrm{R} 12.6$ & 0 & Dr Foster \\
\hline \multirow[t]{6}{*}{24} & 6 & Others & E1.11.1 & N.2 & scientists \\
\hline & & agree & L1.6 & N.5 & agree \\
\hline & & her & $\mathrm{R} 12.6$ & M.1 & $\rightarrow$ She $\rightarrow$ Dr Foster \\
\hline & & think & L1.6 & N.1 & think \\
\hline & & rise & $\mathrm{L} 1.9$ & N.2 & rise \\
\hline & & $\begin{array}{l}\text { severe storms, floods, } \\
\text { droughts, }\end{array}$ & $\mathrm{L} 2.6$ & N.1 & catastrophe \\
\hline \multirow[t]{2}{*}{$\begin{array}{l}\text { Sentence } \\
\text { number }\end{array}$} & $\begin{array}{l}\text { No. of } \\
\text { ties }\end{array}$ & Cohesion item & Type & Distance & Presupposed item \\
\hline & & $\begin{array}{c}\text { famines, the spread of } \\
\text { diseases, the } \\
\text { disappearance of species }\end{array}$ & & & \\
\hline \multirow[t]{8}{*}{25} & 8 & On the other hand & $\mathrm{C} 15.2$ & 0 & (S.24) \\
\hline & & are opposed to & L5 & 0 & agree \\
\hline & & this & $\mathrm{R} 21.6$ & 0 & $(\mathrm{~S} .24)$ \\
\hline & & view & L4.6 & 0 & there may be...of species \\
\hline & & believe & L5 & 0 & think \\
\hline & & levels & L1.6 & N.10 & levels \\
\hline & & carbon dioxide & L1.6 & N.6 & carbon dioxide \\
\hline & & in the air & L2.6 & N.8 & in the atmosphere \\
\hline \multirow[t]{4}{*}{26} & 4 & They & R14.6 & 0 & those, like George Hambley \\
\hline & & predict & L1.6 & N.1 & predict \\
\hline & & warming & L1.6 & N.5 & warming \\
\hline & & $\begin{array}{l}\text { bad environmental } \\
\text { consequences }\end{array}$ & L5 & N.1 & $\begin{array}{l}\text { severe storms, floods, droughts, famines, the } \\
\text { spread of diseases, the disappearance of species }\end{array}$ \\
\hline \multirow[t]{3}{*}{27} & 3 & In fact & $\mathrm{C} 22$ & 0 & (S.26) \\
\hline & & Hambley & L1.6 & N.1 & George Hambley \\
\hline & & carbon dioxide & L1.6 & N.1 & carbon dioxide \\
\hline 28 & 1 & $\operatorname{It}(2 \times)$ & R13.6 & 0 & More carbon dioxide \\
\hline \multirow[t]{2}{*}{29} & 2 & Greenhouse gases & L4.7 & N.1 & carbon dioxide \\
\hline & & in the atmosphere & L2.6 & N.3 & in the air \\
\hline \multirow[t]{2}{*}{30} & 4 & amount & L1.6 & N.9 & amount \\
\hline & & carbon dioxide & $\mathrm{L} 2.7$ & 0 & Greenhouse gases \\
\hline \multirow[t]{3}{*}{$\begin{array}{l}\text { Sentence } \\
\text { number }\end{array}$} & $\begin{array}{c}\text { No. of } \\
\text { ties }\end{array}$ & Cohesion item & Type & Distance & Presupposed item \\
\hline & & greenhouse gases & $\mathrm{L} 1.7$ & 0 & Greenhouse gases \\
\hline & & the climate & L1.6 & N.6 & the climate \\
\hline \multirow[t]{2}{*}{31} & 2 & warming $(2 \times)$ & L1.6 & N.3 & warming \\
\hline & & global & L1.6 & N.16 & global \\
\hline 32 & 1 & that & R22.6 & 0 & (S.31) \\
\hline 33 & 1 & Or & C11.3 & 0 & (S.32) \\
\hline
\end{tabular}




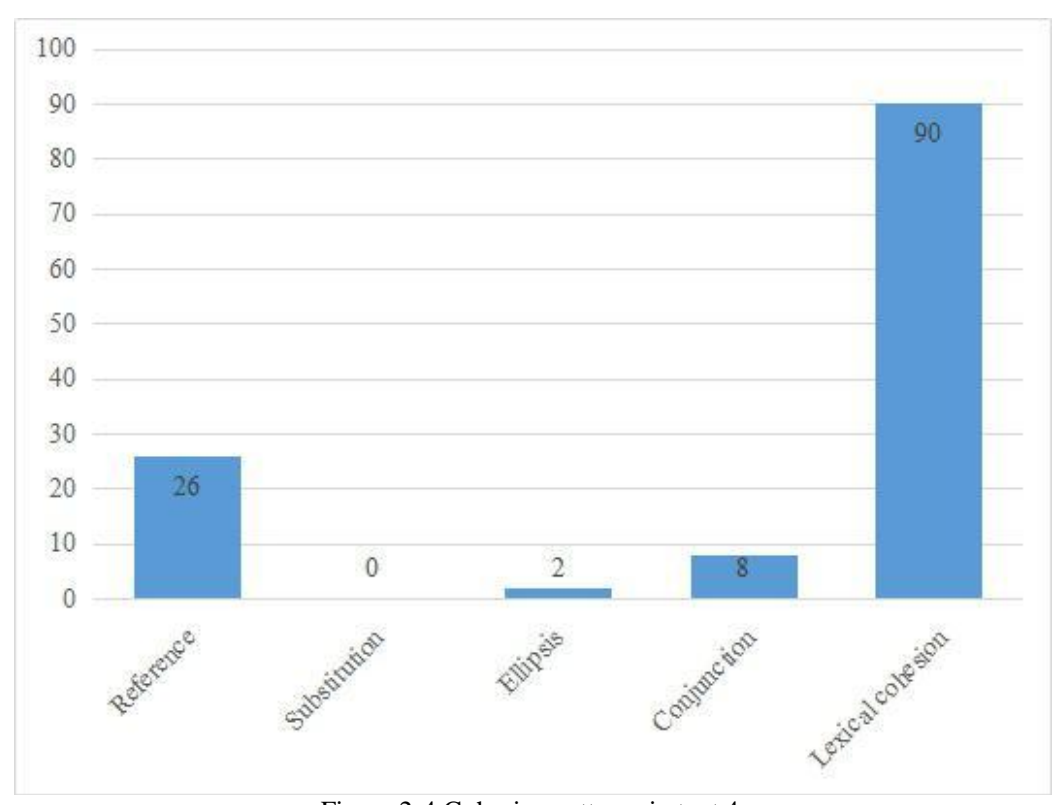

Figure 2-4 Cohesive patterns in text 4

As it can be seen from the above histogram, among the five lexical cohesive devices, lexical cohesion appears 90 times which proved to be the most in the texts while reference secures the second place with 26 occurrences, but far less than the first one. Conjunction and ellipsis are even less, 8 and 2 occurrences respectively. Substitution accounts for nothing without occurring.

TABLE 2-5

THE ANALYSIS OF COHESION IN TEXT 5

\begin{tabular}{|c|c|c|c|c|c|}
\hline $\begin{array}{l}\text { Sentence } \\
\text { number }\end{array}$ & $\begin{array}{c}\text { No. of } \\
\text { ties }\end{array}$ & Cohesion item & Type & Distance & Presupposed item \\
\hline 2 & 2 & $\begin{array}{c}\text { work } \\
\text { the world }\end{array}$ & $\begin{array}{c}\text { L5 } \\
\text { L1.6 }\end{array}$ & $\begin{array}{l}0 \\
0\end{array}$ & $\begin{array}{c}\text { job } \\
\text { the world }\end{array}$ \\
\hline 3 & & working & L1.6 & 0 & work \\
\hline 4 & & job & L5 & 0 & working \\
\hline 5 & & $\begin{array}{l}\text { However } \\
\text { my job } \\
\text { people }\end{array}$ & $\begin{array}{l}\text { L1. } 6 \\
\text { L1.9 }\end{array}$ & $\begin{array}{c}0 \\
0 \\
\text { N.1 }\end{array}$ & $\begin{array}{l}\text { (S.4) } \\
\text { my job } \\
\text { people }\end{array}$ \\
\hline 6 & & $\begin{array}{c}\text { volcanologist } \\
\text { working } \\
\text { Volcano Observatory } \\
\text { (HVO) }\end{array}$ & $\begin{array}{c}\text { L5 } \\
\text { L5 } \\
\text { L1.6 }\end{array}$ & $\begin{array}{l}1 \\
0 \\
0 \\
0\end{array}$ & $\begin{array}{l}\text { volcano } \\
\text { job } \\
\text { volcano }\end{array}$ \\
\hline 7 & & $\begin{array}{c}\text { My job } \\
\text { volcanoes } \\
\text { Hawaii }\end{array}$ & $\begin{array}{l}\text { L1.6 } \\
\text { L1.7 } \\
\text { L1.6 }\end{array}$ & $\begin{array}{c}\mathrm{N} .1 \\
0 \\
0\end{array}$ & $\begin{array}{c}\text { my job } \\
\text { Volcano } \\
\text { Hawaiian }\end{array}$ \\
\hline 8 & & $\begin{array}{l}\text { collected } \\
\text { information } \\
\text { the } \\
\text { volcano }\end{array}$ & $\begin{array}{c}\text { L1.6 } \\
\text { L1.6 } \\
\text { R23.6 } \\
\text { L4.6 }\end{array}$ & $\begin{array}{l}0 \\
0 \\
0 \\
0\end{array}$ & $\begin{array}{c}\text { collecting } \\
\text { information } \\
\text { Mount Kilauea } \\
\text { Mount Kilauea }\end{array}$ \\
\hline 9 & & work & $\mathrm{L} 2.7$ & N.1 & job \\
\hline & & $\begin{array}{l}\text { people } \\
\text { the } \\
\text { lava }\end{array}$ & $\begin{array}{l}\text { L1.6 } \\
\text { R23.6 } \\
\text { L1.6 }\end{array}$ & $\begin{array}{c}\mathrm{N} .3 \\
0 \\
0\end{array}$ & $\begin{array}{c}\text { people } \\
\text { lava from the volcano } \\
\text { lava }\end{array}$ \\
\hline 10 & & $\begin{array}{c}\text { Unfortunately } \\
\text { their } \\
\text { homes }\end{array}$ & $\begin{array}{c}\text { C21.3 } \\
\text { R14.8 } \\
\text { L2.6 }\end{array}$ & $\begin{array}{l}0 \\
0 \\
0\end{array}$ & $\begin{array}{c}\text { (S.9) } \\
\text { people } \\
\text { houses }\end{array}$ \\
\hline $\begin{array}{l}\text { Sentence } \\
\text { number }\end{array}$ & $\begin{array}{l}\text { No. of } \\
\text { ties }\end{array}$ & Cohesion item & Type & Distance & Presupposed item \\
\hline & & $\begin{array}{c}\text { the } \\
\text { way } \\
\text { houses } \\
\text { lava }\end{array}$ & $\begin{array}{l}\text { R23.6 } \\
\text { L2.6 } \\
\text { L1.6 } \\
\text { L1.6 }\end{array}$ & $\begin{array}{l}0 \\
0 \\
0 \\
0\end{array}$ & $\begin{array}{l}\text { the path } \\
\text { path } \\
\text { houses } \\
\text { lava }\end{array}$ \\
\hline 11 & & $\begin{array}{l}\text { boiling } \\
\text { volcano } \\
\text { earth }\end{array}$ & $\begin{array}{c}\text { L5 } \\
\text { L1.7 } \\
\text { L2.6 }\end{array}$ & $\begin{array}{c}0 \\
\text { N.2 } \\
0\end{array}$ & $\begin{array}{l}\text { burned } \\
\text { volcano } \\
\text { ground }\end{array}$ \\
\hline 12 & & $\begin{array}{c}\text { This } \\
\text { because }\end{array}$ & $\begin{array}{l}\text { R21.6 } \\
\text { C33 }\end{array}$ & $\begin{array}{l}0 \\
0\end{array}$ & $\begin{array}{l}\text { (S.11) } \\
(\mathrm{S} .11)\end{array}$ \\
\hline
\end{tabular}




\begin{tabular}{|c|c|c|c|c|c|}
\hline & & $\begin{array}{c}\text { lives } \\
\text { Mount Kilauea } \\
\text { fall } \\
\end{array}$ & $\begin{array}{c}\text { L5 } \\
\text { L1.6 } \\
\text { L2.6 } \\
\end{array}$ & $\begin{array}{c}\text { N.2 } \\
\text { N.4 } \\
0 \\
\end{array}$ & $\begin{array}{c}\text { lives } \\
\text { Mount Kilauea } \\
\text { crashed back }\end{array}$ \\
\hline \multirow[t]{8}{*}{13} & & lava & L5 & N.1 & volcano \\
\hline & & the & $\mathrm{R} 23.6$ & 0 & Mount Kilauea \\
\hline & & mountain & L4.6 & 0 & Mount Kilauea \\
\hline & & causes & L1.6 & N.1 & causes \\
\hline & & damage & L1.6 & N.1 & damage \\
\hline & & path & L2.6 & N.2 & way \\
\hline & & molten & $\mathrm{L} 2.6$ & N.1 & boiling \\
\hline & & rock & L1.6 & 0 & rocks \\
\hline \multirow[t]{3}{*}{14} & & However & $\mathrm{C} 21.3$ & 0 & $(\mathrm{~S} .13)$ \\
\hline & & the & $\mathrm{R} 23.6$ & N.2 & When boiling rock...to earth \\
\hline & & eruption & L1.6 & N.2 & erupts \\
\hline 15 & & Hawaii & L1.6 & N.7 & Hawaii \\
\hline 16 & & worked & L1.9 & N.6 & work \\
\hline 17 & & asleep & L5 & 0 & went to bed \\
\hline \multirow[t]{2}{*}{$\begin{array}{c}\text { Sentence } \\
\text { number }\end{array}$} & $\begin{array}{c}\text { No. of } \\
\text { ties }\end{array}$ & Cohesion item & Type & Distance & Presupposed item \\
\hline & & bed & L1.6 & 0 & bed \\
\hline 18 & & Hawaii & L1.6 & N.2 & Hawaii \\
\hline \multirow[t]{3}{*}{19} & & sleep & L5 & N.1 & asleep \\
\hline & & suddenly & L1.6 & N.1 & suddenly \\
\hline & & bedroom & L5 & N.1 & bed \\
\hline \multirow[t]{3}{*}{20} & & the house & L5 & 0 & my bedroom \\
\hline & & the back garden & L5 & 0 & my bedroom \\
\hline & & Mount Kilauea & L1.6 & N.7 & Mount Kilauea \\
\hline \multirow[t]{7}{*}{21} & & There & R22.7 & 0 & the back garden \\
\hline & & eruption & L1. 6 & N.6 & eruption \\
\hline & & the & $\mathrm{R} 23.6$ & 0 & Mount Kilauea \\
\hline & & mountain & L4.6 & 0 & Mount Kilauea \\
\hline & & red hot & L2.6 & N.7 & molten \\
\hline & & lava & L1.6 & N.7 & lava \\
\hline & & fountaining & $\mathrm{L} 2.6$ & N.9 & erupts \\
\hline \multirow[t]{3}{*}{22} & & It & R13.6 & 0 & an eruption \\
\hline & & fantastic & $\mathrm{R} 2.6$ & N.7 & exciting \\
\hline & & sight & L1.6 & N.7 & sight \\
\hline \multirow[t]{4}{*}{23} & & The day after this eruption & L5 & N.7 & in the second week after... \\
\hline & & this & $\mathrm{R} 21.6$ & N.1 & an eruption \\
\hline & & eruption & L1.6 & N.1 & eruption \\
\hline & & look at & $\mathrm{L} 2.6$ & N.2 & see \\
\hline \multirow[t]{3}{*}{24} & & scientists & L1.6 & N.15 & scientists \\
\hline & & the & $\mathrm{R} 23.6$ & N.2 & the mountain \\
\hline & & mountain & L1.6 & N.2 & mountain \\
\hline \multirow[t]{4}{*}{$\begin{array}{l}\text { Sentence } \\
\text { number }\end{array}$} & $\begin{array}{l}\text { No. of } \\
\text { ties }\end{array}$ & Cohesion item & Type & Distance & Presupposed item \\
\hline & & close & L1.6 & 0 & closer \\
\hline & & the & $\mathrm{R} 23.6$ & 0 & this eruption \\
\hline & & eruption & L1. 6 & 0 & eruption \\
\hline \multirow[t]{4}{*}{25} & & earlier & L1.9 & N.8 & early \\
\hline & & collected & L1.6 & N.16 & collected \\
\hline & & observatory & L1.6 & N.18 & the Hawaiian Volcano Observatory \\
\hline & & closer & L1.6 & 0 & close \\
\hline \multirow[t]{2}{*}{26} & & three & R34.7 & N.1 & Two other scientists and I \\
\hline & & looked & $\mathrm{L} 1.9$ & N.2 & looked \\
\hline \multirow[t]{3}{*}{27} & & suits & $\mathrm{L} 2.6$ & N.1 & clothes \\
\hline & & covered & L1.9 & N.16 & covered \\
\hline & & special & L1.6 & N.1 & special \\
\hline \multirow[t]{8}{*}{28} & & these & R21.6 & 0 & suits \\
\hline & & suits & L1.6 & 0 & suits \\
\hline & & way & L1.9 & N.17 & way \\
\hline & & the & R23.6 & N.3 & the crater \\
\hline & & crater & L1.6 & N.3 & crater \\
\hline & & looked & L1.6 & N.4 & looked \\
\hline & & red & L1.6 & N.6 & red \\
\hline & & boiling & L1.6 & N.16 & boiling \\
\hline 29 & & the & R23.6 & N.4 & Two other scientists \\
\hline & & other & R33.9 & N.4 & Two other scientists \\
\hline
\end{tabular}




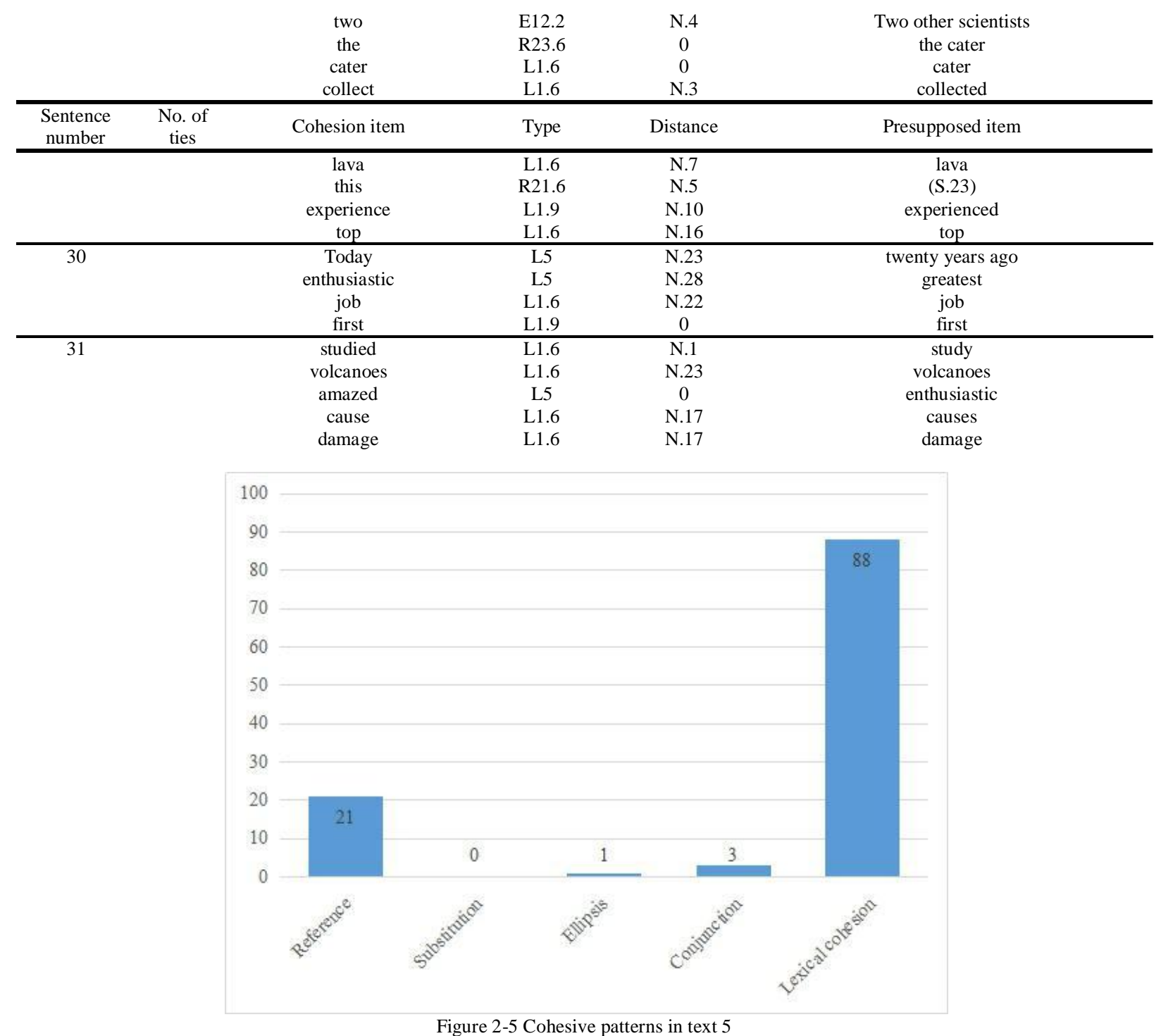

As it can be seen from the above histogram, among the five lexical cohesive devices, lexical cohesion appears 88 times which proved to be the most in the texts while reference secures the second place with 21 occurrences, but far less than the first one. Conjunction and ellipsis are even less, 3 and 1 occurrences respectively. Substitution accounts for nothing without occurring.

\section{ANALYSIS OF LEXICAL COHESION IN THE TEXTS}

After meticulous judging and taking notes of cohesive devices from the 5 texts, we can thus get the total amount of each cohesive device and their contrasts are made into the following table:

TABLE 3-1

FREQUENCY OF COHESIVE DEVICES IN THE Five TeXTS

\begin{tabular}{|c|c|c|c|c|c|}
\hline Cobociur dovice & text 1 & text 2 & text 3 & text 4 & text 5 \\
\hline Corpus & Frequency & Frequency & Frequency & Frequency & Frequency \\
\hline Reference & 41 & 27 & 11 & 26 & 21 \\
\hline substitution & 0 & 1 & 0 & 0 & 0 \\
\hline Ellipsis & 2 & 0 & 4 & 2 & 1 \\
\hline Conjunction & 8 & 5 & 6 & 8 & 3 \\
\hline Lexical cohesion & 109 & 66 & 81 & 90 & 88 \\
\hline
\end{tabular}

From the table above it is obvious that in texts in New Senior English for China Student's Book 5 and 6, the use of lexical cohesion accounts for $72.3 \%$ of the total, the distribution of reference is $21 \%$. Other cohesive devices are adopted less in the texts. For example, substitution and ellipsis are barely used. 
A conclusion can be drawn that lexical cohesion plays a significant role in textual cohesion, especially in written texts. Therefore, a further study on lexical cohesion analysis of these texts is conducted, as is shown in table 6, 7, 8, 9 and 10 .

TABLE 3-2

THE ANALYSIS OF LEXICAL COHESION IN TEXT 1

\begin{tabular}{|c|c|c|c|c|}
\hline & identical & inclusive & exclusive & unrelated \\
\hline Same item & 50 & 5 & 5 & 2 \\
\hline $\begin{array}{c}\text { Synonym or near synonym(incl } \\
\text { hyponym) }\end{array}$ & 8 & 1 & 0 & 0 \\
\hline Superordinate & 3 & 0 & 1 & 0 \\
\hline 'General' item & 1 & 0 & 0 & 0 \\
\hline Collocation & - & - & - & - \\
\hline
\end{tabular}

TABLE 3-3

THE ANALYSIS OF LeXICAL COHESION IN THE TEXT 2

\begin{tabular}{|c|c|c|c|c|}
\hline & inclusive & exclusive & unrelated & \\
\hline Same item & identical & 2 & 2 & 5 \\
\hline $\begin{array}{c}\text { Synonym or near synonym(incl } \\
\text { hyponym) }\end{array}$ & 6 & 0 & 0 & 0 \\
\hline Superordinate & 0 & 0 & 0 & 0 \\
\hline 'General' item & 2 & 0 & 0 & 0 \\
\hline Collocation & - & - & - & \\
\hline
\end{tabular}

TABLE 3-4

THE ANALysis OF LEXICAL COHESION IN THE TEXT 3

\begin{tabular}{|c|c|c|c|c|}
\hline & identical & inclusive & exclusive & unrelated \\
\hline Same item & 44 & 3 & 3 & 2 \\
\hline $\begin{array}{c}\text { Synonym or near synonym(incl } \\
\text { hyponym) }\end{array}$ & 2 & 0 & 0 & 0 \\
\hline Superordinate & 0 & 0 & 0 & 0 \\
\hline 'General' item & 1 & 0 & 0 & 0 \\
\hline Collocation & - & - & - & \\
\hline
\end{tabular}

TABLE 3-5

THE ANALYSIS OF LEXICAL COHESION IN THE TEXT 4

\begin{tabular}{|c|c|c|c|c|}
\hline & identical & inclusive & exclusive & unrelated \\
\hline Same item & 51 & 3 & 2 & 2 \\
\hline $\begin{array}{c}\text { Synonym or near synonym(incl } \\
\text { hyponym) }\end{array}$ & 8 & 1 & 1 & 1 \\
\hline Superordinate & 0 & 0 & 0 & 0 \\
\hline 'General' item & 4 & 1 & 0 & 0 \\
\hline Collocation & - & - & - & \\
\hline
\end{tabular}

TABLE 3-6

THE ANALYSIS OF LEXICAL COHESION IN THE TEXT 5

\begin{tabular}{|l|l|l|l|l|l|}
\hline & identical & inclusive & exclusive & unrelated \\
\hline Same item & 48 & 2 & 0 & 8 & 0 \\
\hline $\begin{array}{l}\text { Synonym or near synonym(incl } \\
\text { hyponym) }\end{array}$ & 10 & 1 & 0 & \\
\hline Superordinate & 0 & 0 & & 0 & \\
\hline 'General' item & 3 & - & - & & \\
\hline Collocation & - & - & & \\
\hline
\end{tabular}




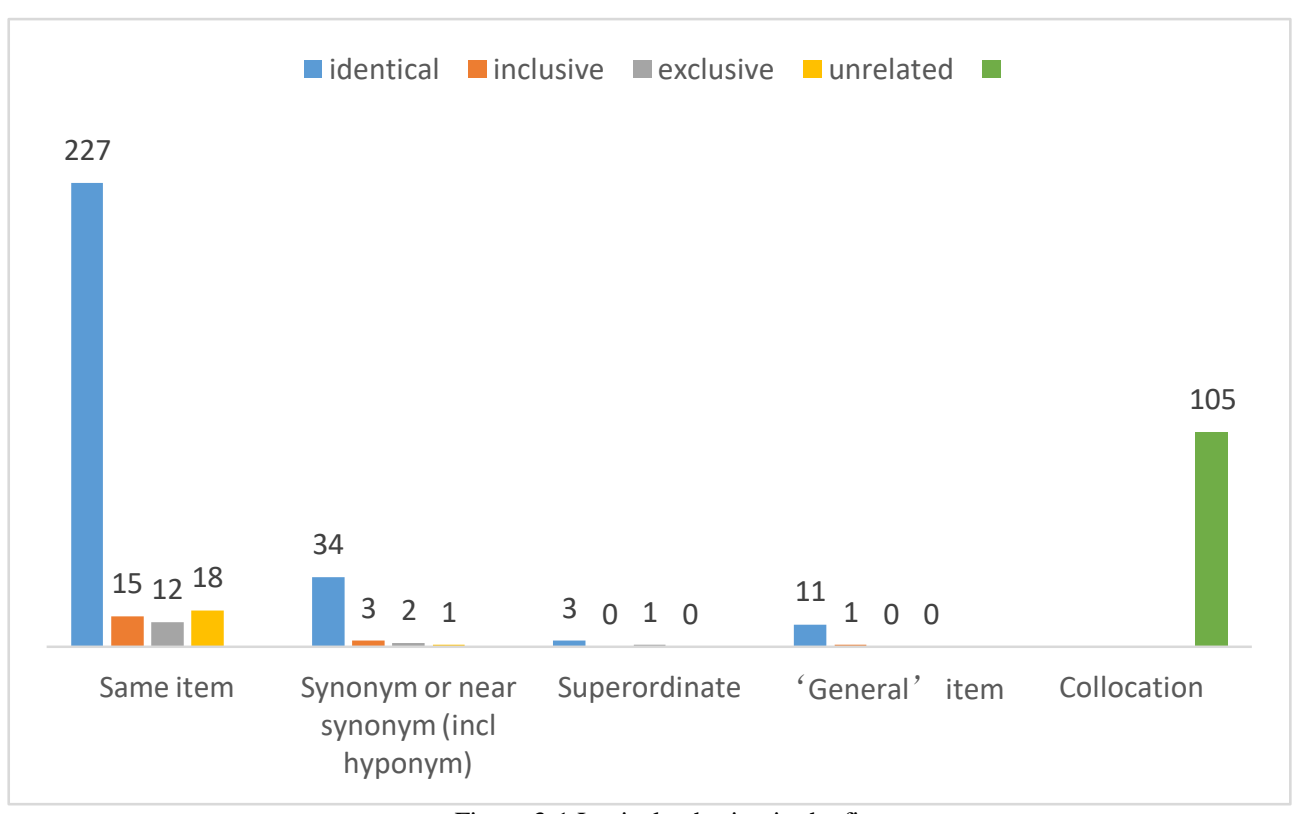

Figure 3-1 Lexical cohesion in the five texts

As it can be seen from the above histogram, among the five lexical cohesive devices, same item having reference that is identical occurs 227 times which proved to be the most in the texts while collocation secure the second place with 105 occurrences; synonym or near synonym including hyponym occurs 34 times on the third place, but far less than the first two. Same item having reference that is unrelated, inclusive and exclusive are even less, 18, 15 and 12 occurrences respectively. 'General item' having reference that is identical takes occurrences 11 times in the whole five texts. The rest of lexical cohesive devices accounts for almost nothing even with some of them never occurring.

\section{CONCLUSION}

Probably the most striking feature of the texts is their lexical explicitness. This comes out especially in the reliance on lexical cohesion. The writer, we feel, does not trust the reader to do much coherence-construction, but seems to aim to enlarge the intended reader's vocabulary by providing various new ways of expressing the same item. Given that the texts are from school textbooks for Chinese teenagers, this is perhaps understandable: the student readers almost certainly do not have the ability to make much sense of a fairly complex text whose coherence may largely depends on substitution or ellipsis.

In fact, these texts are also characterized as full of grammatical structures, the structural relations, especially within the sentence. Though cohesive relations may be found as well within a sentence as between sentences, cohesive ties between sentences stand out more clearly because they are the only source of texture. To distinguish one type of text from another, cohesive ties makes it possible to transcend the boundaries of the clause- that is, the domain of the highest-ranking grammatical unit (Halliday, 2004). However, this paper focuses on the analysis of non-structure cohesion across sentences, so the distinctive grammatical structure between the sentences of these texts is not described.

\section{REFERENCES}

[1] Halliday, M.A.K. \& Hasan, R. (1976). Cohesion in English. London: Longman, /Beijing, Foreign Language Teaching and Research Press.

[2] Halliday, M. A. K. \& Hasan, R. (1976). An Introduction to Functional Grammar. London: Foreign Language. Teaching and Research Press.

[3] Halliday, M.A.K. and Christian Matthiessen. (2004). An Introduction to Functional Grammar. Great Britain: Hodder Education, /Beijing, Foreign Language Teaching and Research Press.

[4] Hu Zhuanglin, Zhu Yongsheng, Zhang Delu. (1987). A Survey of Systemic-Functional Grammar. Changsha: Hunan Education Press.

[5] Hu Zhuanglin. (2017). Textual Cohesion and Coherence (New Edition). Shanghai: East China University Press.

[6] Huang Guowen. (1988). Essentials of Text Analysis. Changsha: Hunan Education Press.

[7] Thompson, G. (2004). Introducing Functional Grammar. Great Britain: Hodder Education, /Beijing, Foreign Language Teaching and Research Press.

[8] Zhang Delu. (2012). New Developments in the Theory of Discourse Analysis and Its Application. Beijing, Foreign Language Teaching and Research Press 


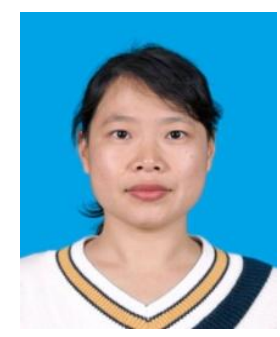

Yuan Zhao was born in Shanxi, China in 1984. She received her master's degree in linguistics from Shanxi Normal University, China in 2021. She is currently a founder of an education institution and also a high school English teacher in the institution. Her research interests include psycholinguistics and senior high school English instruction. 